\title{
A singularly perturbed Dirichlet problem for the Poisson equation in a periodically perforated domain. A functional analytic approach
}

\author{
Paolo Musolino
}

\begin{abstract}
Let $\Omega$ be a sufficiently regular bounded open connected subset of $\mathbb{R}^{n}$ such that $0 \in \Omega$ and that $\mathbb{R}^{n} \backslash \mathrm{cl} \Omega$ is connected. Then we take $\left.\left(q_{11}, \ldots, q_{n n}\right) \in\right] 0,+\infty\left[{ }^{n}\right.$ and $\left.p \in Q \equiv \prod_{j=1}^{n}\right] 0, q_{j j}[$. If $\epsilon$ is a small positive number, then we define the periodically perforated domain $\mathbb{S}\left[\Omega_{p, \epsilon}\right]^{-} \equiv \mathbb{R}^{n} \backslash \cup_{z \in \mathbb{Z}^{n}} \operatorname{cl}\left(p+\epsilon \Omega+\sum_{j=1}^{n}\left(q_{j j} z_{j}\right) e_{j}\right)$, where $\left\{e_{1}, \ldots, e_{n}\right\}$ is the canonical basis of $\mathbb{R}^{n}$. For $\epsilon$ small and positive, we introduce a particular Dirichlet problem for the Poisson equation in the set $\mathbb{S}\left[\Omega_{p, \epsilon}\right]^{-}$. Namely, we consider a Dirichlet condition on the boundary of the set $p+\epsilon \Omega$, together with a periodicity condition. Then we show real analytic continuation properties of the solution as a function of $\epsilon$, of the Dirichlet datum on $p+\epsilon \partial \Omega$, and of the Poisson datum, around a degenerate triple with $\epsilon=0$.
\end{abstract}

Keywords: Dirichlet problem; singularly perturbed domain; Poisson equation; periodically perforated domain; real analytic continuation in Banach space

MSC: 35J25; 31B10; 45A05; 47H30

\section{Introduction}

In this article, we consider a Dirichlet problem for the Poisson equation in a periodically perforated domain with small holes. We fix once for all a natural number

$$
n \in \mathbb{N} \backslash\{0,1\}
$$

and

$$
\left.\left(q_{11}, \ldots, q_{n n}\right) \in\right] 0,+\infty\left[^{n}\right.
$$

and a periodicity cell

$$
\left.Q \equiv \Pi_{j=1}^{n}\right] 0, q_{j j}[.
$$

Then we denote by meas $(Q)$ the measure of the fundamental cell $Q$, and by $\nu_{Q}$ the outward unit normal to $\partial Q$, where it exists. We denote by $q$ the $n \times n$ diagonal matrix defined by $q \equiv\left(\delta_{i, j} q_{j j}\right)_{i, j=1, \ldots, n}$, where $\delta_{i, j}=1$ if $i=j$ and $\delta_{i, j}=0$ if $i \neq j$, for all $i, j \in\{1, \ldots, n\}$. Clearly, $q \mathbb{Z}^{n} \equiv\left\{q z: z \in \mathbb{Z}^{n}\right\}$ is the set of vertices of a periodic subdivision of $\mathbb{R}^{n}$ corresponding to the fundamental cell $Q$. Let $\left.m \in \mathbb{N} \backslash\{0\}, \alpha \in\right] 0,1[$. Then we take a point $p \in Q$ and a bounded open connected subset $\Omega$ of $\mathbb{R}^{n}$ of class $C^{m, \alpha}$ such that $\Omega^{-} \equiv \mathbb{R}^{n} \backslash \operatorname{cl} \Omega$ is connected and that $0 \in \Omega$. Here 'cl' denotes the closure. If $\epsilon \in \mathbb{R}$, then we set

$$
\Omega_{p, \epsilon} \equiv p+\epsilon \Omega .
$$

Then we take $\epsilon_{0}>0$ such that $\operatorname{cl} \Omega_{p, \epsilon} \subseteq Q$ for $|\epsilon|<\epsilon_{0}$, and we introduce the periodically perforated domain

$$
\mathbb{S}\left[\Omega_{p, \epsilon}\right]^{-} \equiv \mathbb{R}^{n} \backslash \bigcup_{z \in \mathbb{Z}^{n}} \operatorname{cl}\left(\Omega_{p, \epsilon}+q z\right),
$$

for $\epsilon \in]-\epsilon_{0}, \epsilon_{0}\left[\right.$. Let $\rho>0$. Next we fix a function $g_{0}$ in the Schauder space $C^{m, \alpha}(\partial \Omega)$ and a function $f_{0}$ in the Roumieu class $C_{q, \omega, \rho}^{0}\left(\mathbb{R}^{n}\right)_{0}$ of real analytic periodic functions with vanishing integral on $Q$ (see (2.1) and (2.3) in Section 2.) For each triple $(\epsilon, g, f) \in] 0, \epsilon_{0}\left[\times C^{m, \alpha}(\partial \Omega) \times C_{q, \omega, \rho}^{0}\left(\mathbb{R}^{n}\right)_{0}\right.$ we consider the Dirichlet problem

$$
\begin{cases}\Delta u(x)=f(x) & \forall x \in \mathbb{S}\left[\Omega_{p, \epsilon}\right]^{-}, \\ u\left(x+q e_{j}\right)=u(x) & \forall x \in c \mathbb{S}\left[\Omega_{p, \epsilon}\right]^{-}, \quad \forall j \in\{1, \ldots, n\}, \\ u(x)=g((x-p) / \epsilon) & \forall x \in \partial \Omega_{p, \epsilon},\end{cases}
$$

where $\left\{e_{1}, \ldots, e_{n}\right\}$ is the canonical basis of $\mathbb{R}^{n}$. If $\left.(\epsilon, g, f) \in\right] 0, \epsilon_{0}\left[\times C^{m, \alpha}(\partial \Omega) \times C_{q, \omega, \rho}^{0}\left(\mathbb{R}^{n}\right)_{0}\right.$, then problem (1.1) has a unique solution in $C^{m, \alpha}\left(\operatorname{cls}\left[\Omega_{p, \epsilon}\right]^{-}\right)$, and we denote it by $u[\epsilon, g, f](\cdot)$ (cf. Proposition 2.2.)

Then we pose the following questions. 
(i) Let $x$ be fixed in $\mathbb{R}^{n} \backslash\left(p+q \mathbb{Z}^{n}\right)$. What can be said on the map $(\epsilon, g, f) \mapsto u[\epsilon, g, f](x)$ when $(\epsilon, g, f)$ approaches $\left(0, g_{0}, f_{0}\right)$ ?

(ii) What can be said on the map $(\epsilon, g, f) \mapsto \int_{Q \backslash \mathrm{c} 1 \Omega_{p, \epsilon}}\left|D_{x} u[\epsilon, g, f](x)\right|^{2} d x$ when $(\epsilon, g, f)$ approaches $\left(0, g_{0}, f_{0}\right)$ ?

(iii) What can be said on the map $(\epsilon, g, f) \mapsto \int_{Q \backslash \mathrm{cl} \Omega_{p, \epsilon}} u[\epsilon, g, f](x) d x$ when $(\epsilon, g, f)$ approaches $\left(0, g_{0}, f_{0}\right)$ ?

Questions of this type have long been investigated with the methods of Asymptotic Analysis, which aim at computing, for example, an asymptotic expansion of the value of the solution at a fixed point in terms of the parameter $\epsilon$. Here, we mention, e.g., Ammari and Kang [1, Ch. 5], Kozlov, Maz'ya, and Movchan [13], Maz'ya, Nazarov, and Plamenewskij [26], Ozawa [30], Ward and Keller [36]. We also mention the vast literature of Calculus of Variations and of Homogenization Theory, where the interest is focused on the limiting behaviour as the singular perturbation parameter degenerates (cf. e.g., Cioranescu and Murat $[4,5]$.

Here instead we wish to characterize the behaviour of $u[\epsilon, g, f](\cdot)$ at $(\epsilon, g, f)=\left(0, g_{0}, f_{0}\right)$ by a different approach. Thus for example, if we consider a certain function, say $F(\epsilon, g, f)$, relative to the solution such as, for example, one of those considered in questions (i)-(iii) above, we would try to prove that $F(\cdot, \cdot, \cdot)$ can be continued real analytically around $(\epsilon, g, f)=\left(0, g_{0}, f_{0}\right)$. We observe that our approach does have its advantages. Indeed, if we know that $F(\epsilon, g, f)$ equals, for $\epsilon>0$, a real analytic operator of the variable $(\epsilon, g, f)$ defined on a whole neighborhood of $\left(0, g_{0}, f_{0}\right)$, then, for example, we could infer the existence of a sequence of real numbers $\left\{a_{j}\right\}_{j=0}^{\infty}$ and of a positive real number $\left.\epsilon^{\prime} \in\right] 0, \epsilon_{0}[$ such that

$$
\left.F\left(\epsilon, g_{0}, f_{0}\right)=\sum_{j=0}^{\infty} a_{j} \epsilon^{j} \quad \forall \epsilon \in\right] 0, \epsilon^{\prime}[
$$

where the power series in the right hand side converges absolutely on the whole of $]-\epsilon^{\prime}, \epsilon^{\prime}[$. Such a project has been carried out by Lanza de Cristoforis in several papers for problems in a bounded domain with a small hole (cf. e.g., Lanza [16, 17, 20, 21].) In the frame of linearized elastostatics, we mention, e.g., Dalla Riva and Lanza $[7,8]$, and for a boundary value problem for the Stokes system in a singularly perturbed exterior domain we refer to Dalla Riva [6]. Instead, for periodic problems, we mention [24, 29], where Dirichlet and nonlinear Robin problems for the Laplace equation have been considered.

As far as problems in periodically perforated domains are concerned, we mention, for instance, Ammari and Kang [1, Ch. 8]. We also observe that boundary value problems in domains with periodic inclusions can be analysed, at least for the two dimensional case, with the method of functional equations. Here we mention, e.g., Castro and Pesetskaya [2], Castro, Pesetskaya, and Rogosin [3], Drygas and Mityushev [10], Mityushev and Adler [28], Rogosin, Dubatovskaya, and Pesetskaya [34]. In connection with doubly periodic problems for composite materials, we mention the monograph of Grigolyuk and Fil'shtinskij [12].

We now briefly outline our strategy. By means of a periodic analog of the Newtonian potential, we can convert problem (1.1) into an auxiliary Dirichlet problem for the Laplace equation. Next we analyze the dependence of the solution of the auxiliary problem upon the triple $(\epsilon, g, f)$ by exploiting the results of [29] on the homogeneous Dirichlet problem and of Lanza [18] on the Newtonian potential in the Roumieu classes, and then we deduce the representation of $u[\epsilon, g, f](\cdot)$ in terms of $\epsilon, g$, and $f$, and we prove our main results.

This article is organized as follows. Section 2 is a section of preliminaries. In Section 3, we formulate the auxiliary Dirichlet problem for the Laplace equation and we show that the solutions of the auxiliary problem depend real analytically on $\epsilon, g$, and $f$. In Section 4, we show that the results of Section 3 can be exploited to prove Theorem 4.1 on the representation of $u[\epsilon, g, f](\cdot)$, Theorems 4.2 and 4.4 on the representation of the energy integral and of the integral of the solution, respectively. At the end of the paper, we have included an Appendix on a slight variant of a result on composition operators of Preciso (cf. Preciso [31, Prop. 4.2.16, p. 51], Preciso [32, Prop. 1.1, p. 101]), which belongs to a different flow of ideas and which accordingly we prefer to discuss separately.

\section{Notation and preliminaries}

We denote the norm on a normed space $\mathcal{X}$ by $\|\cdot\|_{\mathcal{X}}$. Let $\mathcal{X}$ and $\mathcal{Y}$ be normed spaces. We endow the space $\mathcal{X} \times \mathcal{Y}$ with the norm defined by $\|(x, y)\|_{\mathcal{X} \times \mathcal{Y}} \equiv\|x\|_{\mathcal{X}}+\|y\|_{\mathcal{Y}}$ for all $(x, y) \in \mathcal{X} \times \mathcal{Y}$, while we use the Euclidean norm for $\mathbb{R}^{n}$. For standard definitions of Calculus in normed spaces, we refer to Prodi and Ambrosetti [33]. The symbol $\mathbb{N}$ denotes the set of natural numbers including 0. A dot "." denotes the inner product in $\mathbb{R}^{n}$. Let $A$ be a matrix. Then $A_{i j}$ denotes the $(i, j)$-entry of $A$, and the inverse of the matrix $A$ is denoted by $A^{-1}$. Let $\mathbb{D} \subseteq \mathbb{R}^{n}$. Then cld denotes the closure of $\mathbb{D}$ and $\partial \mathbb{D}$ denotes the boundary of $\mathbb{D}$. For all $R>0, x \in \mathbb{R}^{n}$, $x_{j}$ denotes the $j$-th coordinate of $x,|x|$ denotes the Euclidean modulus of $x$ in $\mathbb{R}^{n}$, and $\mathbb{B}_{n}(x, R)$ denotes the 
ball $\left\{y \in \mathbb{R}^{n}:|x-y|<R\right\}$. Let $\Omega$ be an open subset of $\mathbb{R}^{n}$. The space of $m$ times continuously differentiable real-valued functions on $\Omega$ is denoted by $C^{m}(\Omega, \mathbb{R})$, or more simply by $C^{m}(\Omega)$. $\mathcal{D}(\Omega)$ denotes the space of functions of $C^{\infty}(\Omega)$ with compact support. The dual $\mathcal{D}^{\prime}(\Omega)$ denotes the space of distributions in $\Omega$. Let $r \in \mathbb{N} \backslash\{0\}$. Let $f \in\left(C^{m}(\Omega)\right)^{r}$. The $s$-th component of $f$ is denoted $f_{s}$, and $D f$ denotes the Jacobian matrix $\left(\frac{\partial f_{s}}{\partial x_{l}}\right)_{\substack{s=1, \ldots, r \\ l=1, \ldots, n}}$. Let $\eta \equiv\left(\eta_{1}, \ldots, \eta_{n}\right) \in \mathbb{N}^{n},|\eta| \equiv \eta_{1}+\cdots+\eta_{n}$. Then $D^{\eta} f$ denotes $\frac{\partial^{|\eta|} f}{\partial x_{1}^{\eta_{1}} \ldots \partial x_{n}^{\eta_{n}}}$. The subspace of $C^{m}(\Omega)$ of those functions $f$ whose derivatives $D^{\eta} f$ of order $|\eta| \leq m$ can be extended with continuity to cl $\Omega$ is denoted $C^{m}(\mathrm{cl} \Omega)$. The subspace of $C^{m}(\mathrm{cl} \Omega)$ whose functions have $m$-th order derivatives that are Hölder continuous with exponent $\alpha \in] 0,1]$ is denoted $C^{m, \alpha}(\mathrm{cl} \Omega)$ (cf. e.g., Gilbarg and Trudinger [11].) The subspace of $C^{m}(\operatorname{cl} \Omega)$ of those functions $f$ such that $f_{\mid \mathrm{cl}\left(\Omega \cap \mathbb{B}_{n}(0, R)\right)} \in C^{m, \alpha}\left(\operatorname{cl}\left(\Omega \cap \mathbb{B}_{n}(0, R)\right)\right)$ for all $\left.R \in\right] 0,+\infty[$ is denoted $C_{\mathrm{loc}}^{m, \alpha}(\operatorname{cl} \Omega)$. Let $\mathbb{D} \subseteq \mathbb{R}^{r}$. Then $C^{m, \alpha}(\operatorname{cl} \Omega, \mathbb{D})$ denotes $\left\{f \in\left(C^{m, \alpha}(\operatorname{cl} \Omega)\right)^{r}: \quad f(\operatorname{cl} \Omega) \subseteq \mathbb{D}\right\}$.

Now let $\Omega$ be a bounded open subset of $\mathbb{R}^{n}$. Then $C^{m}(\mathrm{cl} \Omega)$ and $C^{m, \alpha}(\operatorname{cl} \Omega)$ are endowed with their usual norm and are well known to be Banach spaces (cf. e.g., Troianiello [35, §1.2.1].) We say that a bounded open subset $\Omega$ of $\mathbb{R}^{n}$ is of class $C^{m}$ or of class $C^{m, \alpha}$, if its closure is a manifold with boundary imbedded in $\mathbb{R}^{n}$ of class $C^{m}$ or $C^{m, \alpha}$, respectively (cf. e.g., Gilbarg and Trudinger [11, §6.2].) We denote by $\nu_{\Omega}$ the outward unit normal to $\partial \Omega$. For standard properties of functions in Schauder spaces, we refer the reader to Gilbarg and Trudinger [11] and to Troianiello [35] (see also Lanza [14, §2, Lem. 3.1, 4.26, Thm. 4.28], Lanza and Rossi [25, $\S 2]$.

If $\mathbb{M}$ is a manifold imbedded in $\mathbb{R}^{n}$ of class $C^{m, \alpha}$, with $\left.\left.m \geq 1, \alpha \in\right] 0,1\right]$, one can define the Schauder spaces also on $\mathbb{M}$ by exploiting the local parametrizations. In particular, one can consider the spaces $C^{k, \alpha}(\partial \Omega)$ on $\partial \Omega$ for $0 \leq k \leq m$ with $\Omega$ a bounded open set of class $C^{m, \alpha}$, and the trace operator of $C^{k, \alpha}(\operatorname{cl} \Omega)$ to $C^{k, \alpha}(\partial \Omega)$ is linear and continuous. We denote by $d \sigma$ the area element of a manifold imbedded in $\mathbb{R}^{n}$.

We note that throughout the paper "analytic" means "real analytic". For the definition and properties of real analytic operators, we refer to Prodi and Ambrosetti [33, p. 89]. In particular, we mention that the pointwise product in Schauder spaces is bilinear and continuous, and thus analytic (cf. e.g., Lanza and Rossi [25, p. 141].)

For all bounded open subsets $\Omega$ of $\mathbb{R}^{n}$ and $\rho>0$, we set

$$
C_{\omega, \rho}^{0}(\operatorname{cl} \Omega) \equiv\left\{u \in C^{\infty}(\operatorname{cl} \Omega): \sup _{\beta \in \mathbb{N}^{n}} \frac{\rho^{|\beta|}}{|\beta| !}\left\|D^{\beta} u\right\|_{C^{0}(\operatorname{cl} \Omega)}<+\infty\right\},
$$

and

$$
\|u\|_{C_{\omega, \rho}^{0}(\mathrm{cl} \Omega)} \equiv \sup _{\beta \in \mathbb{N}^{n}} \frac{\rho^{|\beta|}}{|\beta| !}\left\|D^{\beta} u\right\|_{C^{0}(\mathrm{cl} \Omega)} \quad \forall u \in C_{\omega, \rho}^{0}(\mathrm{cl} \Omega),
$$

where $|\beta| \equiv \beta_{1}+\cdots+\beta_{n}$, for all $\beta \equiv\left(\beta_{1}, \ldots, \beta_{n}\right) \in \mathbb{N}^{n}$. As is well known, the Roumieu class $\left(C_{\omega, \rho}^{0}(\operatorname{cl} \Omega), \|\right.$. $\left.\|_{C_{\omega, \rho}^{0}(\mathrm{c} l \Omega)}\right)$ is a Banach space.

A straightforward computation based on the inequality $\left(\begin{array}{c}\beta \\ \alpha\end{array}\right) \leq\left(\begin{array}{l}|\beta| \\ |\alpha|\end{array}\right)$, which holds for $\alpha, \beta \in \mathbb{N}^{n}, 0 \leq \alpha \leq \beta$, shows that the pointwise product is bilinear and continuous from $\left(C_{\omega, \rho}^{0}(\operatorname{cl} \Omega)\right)^{2}$ to $C_{\omega, \rho^{\prime}}^{0}(\operatorname{cl} \Omega)$ for all $\left.\rho^{\prime} \in\right] 0, \rho[$.

Let $\mathbb{D} \subseteq \mathbb{R}^{n}$ be such that $q z+\mathbb{D} \subseteq \mathbb{D}$ for all $z \in \mathbb{Z}^{n}$. We say that a function $f$ from $\mathbb{D}$ to $\mathbb{R}$ is $q$-periodic if $f\left(x+q e_{j}\right)=f(x)$ for all $x \in \mathbb{D}$ and for all $j \in\{1, \ldots, n\}$. If $k \in \mathbb{N}$, then we set

$$
C_{q}^{k}\left(\mathbb{R}^{n}\right) \equiv\left\{u \in C^{k}\left(\mathbb{R}^{n}\right): u \text { is } q \text { - periodic }\right\}
$$

We also set

$$
C_{q}^{\infty}\left(\mathbb{R}^{n}\right) \equiv\left\{u \in C^{\infty}\left(\mathbb{R}^{n}\right): u \text { is } q-\text { periodic }\right\}
$$

Similarly, if $\rho>0$, we set

$$
C_{q, \omega, \rho}^{0}\left(\mathbb{R}^{n}\right) \equiv\left\{u \in C_{q}^{\infty}\left(\mathbb{R}^{n}\right): \sup _{\beta \in \mathbb{N}^{n}} \frac{\rho^{|\beta|}}{|\beta| !}\left\|D^{\beta} u\right\|_{C^{0}(\mathrm{cl} Q)}<+\infty\right\},
$$

and

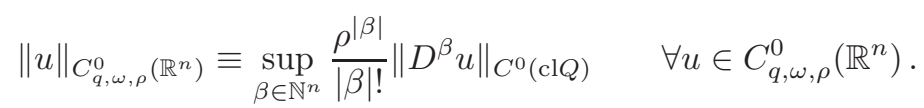

As can be easily seen, the periodic Roumieu class $\left(C_{q, \omega, \rho}^{0}\left(\mathbb{R}^{n}\right),\|\cdot\|_{C_{q, \omega, \rho}^{0}\left(\mathbb{R}^{n}\right)}\right)$ is a Banach space. Obviously, the restriction operator from $C_{q, \omega, \rho}^{0}\left(\mathbb{R}^{n}\right)$ to $C_{\omega, \rho}^{0}(\mathrm{cl} D)$ is linear and continuous for all bounded open subsets $\mathbb{D}$ of $\mathbb{R}^{n}$ and $\rho>0$. Similarly, if $\rho>0$, if $\mathbb{D}$ is a bounded open subset of $\mathbb{R}^{n}$ such that $\operatorname{cl} Q \subseteq \mathbb{D}$, and if $f \in C_{q}^{0}\left(\mathbb{R}^{n}\right)$ is such that $f_{\mid \mathrm{cll}} \in C_{\omega, \rho}^{0}(\mathrm{cl} \mathbb{D})$, then clearly $f \in C_{q, \omega, \rho}^{0}\left(\mathbb{R}^{n}\right)$. 
If $\Omega$ is an open subset of $\left.\left.\mathbb{R}^{n}, k \in \mathbb{N}, \beta \in\right] 0,1\right]$, we set

$$
C_{b}^{k, \beta}(\mathrm{cl} \Omega) \equiv\left\{u \in C^{k, \beta}(\mathrm{cl} \Omega): D^{\gamma} u \text { is bounded } \forall \gamma \in \mathbb{N}^{n} \text { such that }|\gamma| \leq k\right\},
$$

and we endow $C_{b}^{k, \beta}(\mathrm{cl} \Omega)$ with its usual norm

$$
\|u\|_{C_{b}^{k, \beta}(\mathrm{cl} \Omega)} \equiv \sum_{|\gamma| \leq k} \sup _{x \in \operatorname{cl} \Omega}\left|D^{\gamma} u(x)\right|+\sum_{|\gamma|=k}\left|D^{\gamma} u: \operatorname{cl} \Omega\right|_{\beta} \quad \forall u \in C_{b}^{k, \beta}(\operatorname{cl} \Omega),
$$

where $\left|D^{\gamma} u: \operatorname{cl} \Omega\right|_{\beta}$ denotes the $\beta$-Hölder constant of $D^{\gamma} u$.

Next we turn to periodic domains. If $\mathbb{I}$ is an arbitrary subset of $\mathbb{R}^{n}$ such that $\mathrm{clI} \subseteq Q$, then we set

$$
\mathbb{S}[\mathbb{I}] \equiv \bigcup_{z \in \mathbb{Z}^{n}}(q z+\mathbb{I})=q \mathbb{Z}^{n}+\mathbb{I}, \quad \mathbb{S}[\mathbb{I}]^{-} \equiv \mathbb{R}^{n} \backslash \operatorname{cl} \mathbb{S}[\mathbb{I}]
$$

We note that if $\mathbb{R}^{n} \backslash$ clI is connected, then $\mathbb{S}[\mathbb{I}]^{-}$is connected.

If $\mathbb{I}$ is an open subset of $\mathbb{R}^{n}$ such that $\operatorname{cl} \mathbb{I} \subseteq Q$ and if $\left.\left.k \in \mathbb{N}, \beta \in\right] 0,1\right]$, then we set

$$
C_{q}^{k, \beta}(\operatorname{cl} \mathbb{S}[\mathbb{I}]) \equiv\left\{u \in C_{b}^{k, \beta}(\operatorname{cl} \mathbb{S}[\mathbb{I}]): u \text { is } q-\text { periodic }\right\}
$$

which we regard as a Banach subspace of $C_{b}^{k, \beta}(\operatorname{clS}[\mathbb{I}])$, and

$$
C_{q}^{k, \beta}\left(\operatorname{cl} \mathbb{S}[\mathbb{I}]^{-}\right) \equiv\left\{u \in C_{b}^{k, \beta}\left(\operatorname{cl} \mathbb{S}[\mathbb{I}]^{-}\right): u \text { is } q-\text { periodic }\right\}
$$

which we regard as a Banach subspace of $C_{b}^{k, \beta}\left(\mathrm{cl} \mathbb{S}[\mathbb{I}]^{-}\right)$.

The Laplace operator is well known to have a $\{0\}$-analog of a $q$-periodic fundamental solution, i.e., a $q$-periodic tempered distribution $S_{q, n}$ such that

$$
\Delta S_{q, n}=\sum_{z \in \mathbb{Z}^{n}} \delta_{q z}-\frac{1}{\operatorname{meas}(Q)}
$$

where $\delta_{q z}$ denotes the Dirac measure with mass in $q z$ (cf. e.g., $[22, \S 3]$.) As is well known, $S_{q, n}$ is determined up to an additive constant, and we can take

$$
S_{q, n}(x)=-\sum_{z \in \mathbb{Z}^{n} \backslash\{0\}} \frac{1}{\operatorname{meas}(Q) 4 \pi^{2}\left|q^{-1} z\right|^{2}} e^{2 \pi i\left(q^{-1} z\right) \cdot x} \quad \text { in } \mathcal{D}^{\prime}\left(\mathbb{R}^{n}\right)
$$

(cf. e.g., Ammari and Kang [1, p. 53], $[22, \S 3]$. ) Clearly $S_{q, n}$ is even. Moreover, $S_{q, n}$ is real analytic in $\mathbb{R}^{n} \backslash q \mathbb{Z}^{n}$ and is locally integrable in $\mathbb{R}^{n}$.

Let $S_{n}$ be the function from $\mathbb{R}^{n} \backslash\{0\}$ to $\mathbb{R}$ defined by

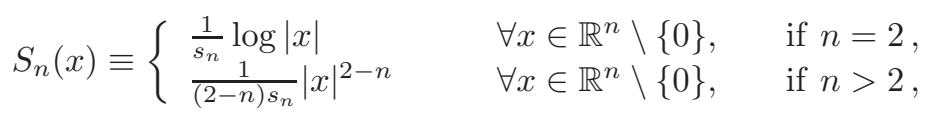

where $s_{n}$ denotes the $(n-1)$ dimensional measure of $\partial \mathbb{B}_{n} . S_{n}$ is well-known to be the fundamental solution of the Laplace operator.

Then the function $S_{q, n}-S_{n}$ is analytic in $\left(\mathbb{R}^{n} \backslash q \mathbb{Z}^{n}\right) \cup\{0\}$ (cf. e.g., Ammari and Kang [1, Lemma 2.39, p. 54], [22, §3].) Then we find convenient to set

$$
R_{n} \equiv S_{q, n}-S_{n} \quad \text { in }\left(\mathbb{R}^{n} \backslash q \mathbb{Z}^{n}\right) \cup\{0\} .
$$

We now introduce the periodic simple layer potential. Let $\alpha \in] 0,1[, m \in \mathbb{N} \backslash\{0\}$. Let $\mathbb{I}$ be a bounded open subset of $\mathbb{R}^{n}$ of class $C^{m, \alpha}$ such that clII $\subseteq Q$. If $\mu \in C^{0, \alpha}(\partial \mathbb{I})$, we set

$$
v_{q}[\partial \mathbb{I}, \mu](x) \equiv \int_{\partial \mathbb{I}} S_{q, n}(x-y) \mu(y) d \sigma_{y} \quad \forall x \in \mathbb{R}^{n} .
$$

As is well known, if $\mu \in C^{m-1, \alpha}(\partial \mathbb{I})$, then the function $v_{q}^{+}[\partial \mathbb{I}, \mu] \equiv v_{q}[\partial \mathbb{I}, \mu]_{\mid \operatorname{cl}[\mathbb{I}]}$ belongs to $C_{q}^{m, \alpha}(\operatorname{cl} \mathbb{S}[\mathbb{I}])$, and the function $v_{q}^{-}[\partial \mathbb{I}, \mu] \equiv v_{q}[\partial \mathbb{I}, \mu]_{\mid c \mathbb{S}[\mathbb{I}]^{-}}$belongs to $C_{q}^{m, \alpha}\left(\operatorname{cl} \mathbb{S}[\mathbb{I}]^{-}\right)$. Similarly, we introduce the periodic double layer potential. If $\mu \in C^{0, \alpha}(\partial \mathbb{I})$, we set

$$
w_{q}[\partial \mathbb{I}, \mu](x) \equiv-\int_{\partial \mathbb{I}}\left(D S_{q, n}(x-y)\right) \nu_{\mathbb{I}}(y) \mu(y) d \sigma_{y} \quad \forall x \in \mathbb{R}^{n} .
$$


If $\mu \in C^{m, \alpha}(\partial \mathbb{I})$, then the restriction $w_{q}[\partial \mathbb{I}, \mu]_{\mid \mathbb{S}[\mathbb{I}]}$ can be extended uniquely to an element $w_{q}^{+}[\partial \mathbb{I}, \mu]$ of $C_{q}^{m, \alpha}(\operatorname{cl} \mathbb{S}[\mathbb{I}])$, and the restriction $w_{q}[\partial \mathbb{I}, \mu]_{\mid \mathbb{S}[\mathbb{I}]^{-}}$can be extended uniquely to an element $w_{q}^{-}[\partial \mathbb{I}, \mu]$ of $C_{q}^{m, \alpha}\left(\operatorname{cl} \mathbb{S}[\mathbb{I}]^{-}\right)$, and we have $w_{q}^{ \pm}[\partial \mathbb{I}, \mu]= \pm \frac{1}{2} \mu+w_{q}[\partial \mathbb{I}, \mu]$ on $\partial \mathbb{I}$. Moreover, if $\mu \in C^{0, \alpha}(\partial \mathbb{I})$, then we have

$$
w_{q}[\partial \mathbb{I}, \mu](x)=-\sum_{j=1}^{n} \frac{\partial}{\partial x_{j}} \int_{\partial \mathbb{I}} S_{q, n}(x-y)\left(\nu_{\mathbb{I}}(y)\right)_{j} \mu(y) d \sigma_{y},
$$

for all $x \in \mathbb{R}^{n} \backslash \partial \mathbb{S}[\mathbb{I}]$ (cf. e.g., $[22, \S 3]$.)

As we have done for the periodic layer potentials, we now introduce a periodic analog of the Newtonian potential. If $f \in C_{q}^{0}\left(\mathbb{R}^{n}\right)$, then we set

$$
P_{q}[f](x) \equiv \int_{Q} S_{q, n}(x-y) f(y) d y \quad \forall x \in \mathbb{R}^{n} .
$$

Clearly, $P_{q}[f]$ is a $q$-periodic function on $\mathbb{R}^{n}$.

In the following Theorem, we collect some elementary properties of the periodic Newtonian potential. A proof can be effected by splitting $S_{q, n}$ into the sum of $S_{n}$ and $R_{n}$, and by exploiting the results of Lanza [18] on the (classical) Newtonian potential in the Roumieu classes and standard properties of integral operators with real analytic kernels and with no singularity (cf. e.g., [23, §3] and [9].)

Theorem 2.1. The following statements hold.

(i) Let $\beta \in] 0,1]$. Let $f \in C_{q}^{0, \beta}\left(\mathbb{R}^{n}\right)$. Then $P_{q}[f] \in C_{q}^{2}\left(\mathbb{R}^{n}\right)$ and

$$
\Delta P_{q}[f](x)=f(x)-\frac{1}{\operatorname{meas}(Q)} \int_{Q} f(y) d y \quad \forall x \in \mathbb{R}^{n} .
$$

(ii) Let $\rho>0$. Then there exists $\left.\left.\rho^{\prime} \in\right] 0, \rho\right]$ such that $P_{q}[f] \in C_{q, \omega, \rho^{\prime}}^{0}\left(\mathbb{R}^{n}\right)$ for all $f \in C_{q, \omega, \rho}^{0}\left(\mathbb{R}^{n}\right)$ and such that $P_{q}[\cdot]$ is linear and continuous from $C_{q, \omega, \rho}^{0}\left(\mathbb{R}^{n}\right)$ to $C_{q, \omega, \rho^{\prime}}^{0}\left(\mathbb{R}^{n}\right)$.

Let $m \in \mathbb{N} \backslash\{0\}, \alpha \in] 0,1\left[\right.$. If $\Omega$ is a bounded open subset of $\mathbb{R}^{n}$ of class $C^{m, \alpha}$, we find convenient to set

$$
C^{m, \alpha}(\partial \Omega)_{0} \equiv\left\{\phi \in C^{m, \alpha}(\partial \Omega): \int_{\partial \Omega} \phi d \sigma=0\right\}
$$

If $\rho>0$, we also set

$$
C_{q, \omega, \rho}^{0}\left(\mathbb{R}^{n}\right)_{0} \equiv\left\{f \in C_{q, \omega, \rho}^{0}\left(\mathbb{R}^{n}\right): \int_{Q} f d x=0\right\} .
$$

As the following Proposition shows, a periodic Dirichlet boundary value problem for the Poisson equation in the perforated domain $\mathbb{S}[\mathbb{I}]^{-}$has a unique solution in $C_{q}^{m, \alpha}\left(\operatorname{clS}[\mathbb{I}]^{-}\right)$, which can be represented as the sum of a periodic double layer potential, of a costant, and of a periodic Newtonian potential.

Proposition 2.2. Let $m \in \mathbb{N} \backslash\{0\}, \alpha \in] 0,1\left[\right.$. Let $\rho>0$. Let $\mathbb{I}$ be a bounded connected open subset of $\mathbb{R}^{n}$ of class $C^{m, \alpha}$ such that $\mathbb{R}^{n} \backslash$ clI $i s$ connected and that $\mathrm{clI} \subseteq Q$. Let $\Gamma \in C^{m, \alpha}(\partial \mathbb{I})$. Let $f \in C_{q, \omega, \rho}^{0}\left(\mathbb{R}^{n}\right)_{0}$. Then the following boundary value problem

$$
\begin{cases}\Delta u(x)=f(x) & \forall x \in \mathbb{S}[\mathbb{I}]^{-}, \\ u \text { is } q \text {-periodic in cl } \mathbb{S}[\mathbb{I}]^{-}, & \\ u(x)=\Gamma(x) & \forall x \in \partial \mathbb{I},\end{cases}
$$

has a unique solution $u \in C_{q}^{m, \alpha}\left(\operatorname{cl} \mathbb{S}[\mathbb{I}]^{-}\right)$. Moreover,

$$
u(x)=w_{q}^{-}[\partial \mathbb{I}, \mu](x)+\xi+P_{q}[f](x) \quad \forall x \in c \mathbb{S}[\mathbb{I}]^{-},
$$

where $(\mu, \xi)$ is the unique solution in $C^{m, \alpha}(\partial \mathbb{I})_{0} \times \mathbb{R}$ of the following integral equation

$$
-\frac{1}{2} \mu(x)+w_{q}[\partial \mathbb{I}, \mu](x)+\xi=\Gamma(x)-P_{q}[f](x) \quad \forall x \in \partial \mathbb{I} .
$$

Proof. A proof can be effected by considering the difference $u-P_{q}[f]$ and by solving the corresponding homogeneous problem (cf. [29, §2] and Theorem 2.1.) 


\section{Formulation and analysis of an auxiliary problem}

We shall consider the following assumptions for some $\alpha \in] 0,1[$ and for some natural $m \geq 1$.

Let $\Omega$ be a bounded connected open subset of $\mathbb{R}^{n}$ of class $C^{m, \alpha}$

such that $\mathbb{R}^{n} \backslash \operatorname{cl} \Omega$ is connected and that $0 \in \Omega$.

Let $p \in Q$.

If $\epsilon \in \mathbb{R}$ and (3.1) holds, we set

$$
\Omega_{p, \epsilon} \equiv p+\epsilon \Omega .
$$

Now let $\epsilon_{0}$ be such that

$$
\left.\epsilon_{0}>0 \quad \text { and } \quad c l \Omega_{p, \epsilon} \subseteq Q \quad \forall \epsilon \in\right]-\epsilon_{0}, \epsilon_{0}[.
$$

A simple topological argument shows that if (3.1) holds, then $\mathbb{S}\left[\Omega_{p, \epsilon}\right]^{-}$is connected, for all $\left.\epsilon \in\right]-\epsilon_{0}, \epsilon_{0}[$. We also note that

$$
\nu_{\Omega_{p, \epsilon}}(p+\epsilon t)=\operatorname{sgn}(\epsilon) \nu_{\Omega}(t) \quad \forall t \in \partial \Omega,
$$

for all $\epsilon \epsilon]-\epsilon_{0}, \epsilon_{0}[\backslash\{0\}$, where $\operatorname{sgn}(\epsilon)=1$ if $\epsilon>0, \operatorname{sgn}(\epsilon)=-1$ if $\epsilon<0$. Let $\rho>0$. Then we shall consider also the following assumptions.

$$
\begin{aligned}
& \text { Let } g_{0} \in C^{m, \alpha}(\partial \Omega) . \\
& \text { Let } f_{0} \in C_{q, \omega, \rho}^{0}\left(\mathbb{R}^{n}\right)_{0} .
\end{aligned}
$$

If $(\epsilon, g, f) \in] 0, \epsilon_{0}\left[\times C^{m, \alpha}(\partial \Omega) \times C_{q, \omega, \rho}^{0}\left(\mathbb{R}^{n}\right)_{0}\right.$, then we denote by $u[\epsilon, g, f]$ the unique solution in $C_{q}^{m, \alpha}\left(\operatorname{cl} \mathbb{S}\left[\Omega_{p, \epsilon}\right]^{-}\right)$ of problem (1.1), and by $u_{\#}[\epsilon, g, f]$ the unique solution in $C_{q}^{m, \alpha}\left(\operatorname{clS}\left[\Omega_{p, \epsilon}\right]^{-}\right)$of the following auxiliary boundary value problem

$$
\begin{cases}\Delta u(x)=0 & \forall x \in \mathbb{S}\left[\Omega_{p, \epsilon}\right]^{-}, \\ u \text { is q-periodic in } \operatorname{cl}\left[\Omega_{p, \epsilon}\right]^{-}, & \\ u(x)=g((x-p) / \epsilon)-P_{q}[f](x) & \forall x \in \partial \Omega_{p, \epsilon} .\end{cases}
$$

Clearly,

$$
u[\epsilon, g, f]=u_{\#}[\epsilon, g, f]+P_{q}[f] \quad \text { on } c \mathbb{S}\left[\Omega_{p, \epsilon}\right]^{-} .
$$

Let $\left.f \in C_{q, \omega, \rho}^{0}\left(\mathbb{R}^{n}\right), \epsilon \in\right] 0, \epsilon_{0}[$. We note that

$$
P_{q}[f](x)=P_{q}[f](p+\epsilon((x-p) / \epsilon)) \quad \forall x \in \partial \Omega_{p, \epsilon} .
$$

Accordingly, the Dirichlet condition in problem (3.5) can be rewritten as

$$
u(x)=\left(g-P_{q}[f] \circ\left(p+\epsilon \operatorname{id}_{\partial \Omega}\right)\right)((x-p) / \epsilon) \quad \forall x \in \partial \Omega_{p, \epsilon},
$$

where id $\partial \Omega$ denotes the identity map in $\partial \Omega$. As a consequence, in order to study the dependence of $u_{\#}[\epsilon, g, f]$ upon $(\epsilon, g, f)$ around $\left(0, g_{0}, f_{0}\right)$, we can exploit the results of [29], concerning the dependence of the solution of the Dirichlet problem for the Laplace equation upon $\epsilon$ and the Dirichlet datum. In order to do so, we need to study the regularity of the map from $]-\epsilon_{0}, \epsilon_{0}\left[\times C_{q, \omega, \rho}^{0}\left(\mathbb{R}^{n}\right)\right.$ to $C^{m, \alpha}(\partial \Omega)$ which takes $(\epsilon, f)$ to the function $P_{q}[f] \circ\left(p+\epsilon \operatorname{id}_{\partial \Omega}\right)$.

Lemma 3.1. Let $m \in \mathbb{N} \backslash\{0\}, \alpha \in] 0,1\left[\right.$. Let $\rho>0$. Let (3.1)-(3.4) hold. Let $\operatorname{id}_{\partial \Omega}$, $\operatorname{id}_{\mathrm{c} \Omega \Omega}$ denote the identity map in $\partial \Omega$ and in $\mathrm{cl} \Omega$, respectively. Then the following statements hold.

(i) The map from $]-\epsilon_{0}, \epsilon_{0}\left[\times C_{q, \omega, \rho}^{0}\left(\mathbb{R}^{n}\right)\right.$ to $C^{m, \alpha}(\mathrm{cl} \Omega)$ which takes $(\epsilon, f)$ to $P_{q}[f] \circ\left(p+\epsilon \mathrm{id}_{\mathrm{c} \Omega}\right)$ is real analytic.

(ii) The map from $]-\epsilon_{0}, \epsilon_{0}\left[\times C_{q, \omega, \rho}^{0}\left(\mathbb{R}^{n}\right)\right.$ to $C^{m, \alpha}(\partial \Omega)$ which takes $(\epsilon, f)$ to $P_{q}[f] \circ\left(p+\epsilon \operatorname{id}_{\partial \Omega}\right)$ is real analytic.

Proof. We first prove statement (i). By Theorem 2.1 (ii), there exists $\left.\left.\rho^{\prime} \in\right] 0, \rho\right]$ such that the linear map from $C_{q, \omega, \rho}^{0}\left(\mathbb{R}^{n}\right)$ to $C_{\omega, \rho^{\prime}}^{0}(\operatorname{cl} Q)$ which takes $f$ to $P_{q}[f]_{\mid c l Q}$ is continuous. As a consequence, by Proposition A.1 of the Appendix, we immediately deduce that the map from $]-\epsilon_{0}, \epsilon_{0}\left[\times C_{q, \omega, \rho}^{0}\left(\mathbb{R}^{n}\right)\right.$ to $C^{m, \alpha}(\operatorname{cl} \Omega)$ which takes $(\epsilon, f)$ to $P_{q}[f] \circ\left(p+\epsilon \mathrm{id}_{\mathrm{c} \Omega} \Omega\right)$ is real analytic. By the continuity of the trace operator from $C^{m, \alpha}(\operatorname{cl} \Omega)$ to $C^{m, \alpha}(\partial \Omega)$ and statement (i), we deduce the validity of (ii).

Then we have the following Lemma (cf. [29, §3].) 
Lemma 3.2. Let $m \in \mathbb{N} \backslash\{0\}, \alpha \in] 0,1\left[\right.$. Let $\rho>0$. Let (3.1)-(3.4) hold. Let $\tau_{0}$ be the unique solution in $C^{m-1, \alpha}(\partial \Omega)$ of the following problem

$$
\left\{\begin{array}{l}
-\frac{1}{2} \tau(t)+\int_{\partial \Omega}\left(D S_{n}(t-s)\right) \nu_{\Omega}(t) \tau(s) d \sigma_{s}=0 \quad \forall t \in \partial \Omega \\
\int_{\partial \Omega} \tau d \sigma=1
\end{array}\right.
$$

Then equation

$$
-\frac{1}{2} \theta(t)-\int_{\partial \Omega}\left(D S_{n}(t-s)\right) \nu_{\Omega}(s) \theta(s) d \sigma_{s}+\xi=g_{0}(t)-P_{q}\left[f_{0}\right](p) \quad \forall t \in \partial \Omega,
$$

which we call the limiting equation, has a unique solution in $C^{m, \alpha}(\partial \Omega)_{0} \times \mathbb{R}$, which we denote by $(\tilde{\theta}, \tilde{\xi})$. Moreover,

$$
\tilde{\xi}=\int_{\partial \Omega} g_{0} \tau_{0} d \sigma-P_{q}\left[f_{0}\right](p)
$$

and the function $\tilde{u} \in C_{\mathrm{loc}}^{m, \alpha}\left(\mathbb{R}^{n} \backslash \mathrm{cl} \Omega\right)$, defined by

$$
\tilde{u}(t) \equiv-\int_{\partial \Omega}\left(D S_{n}(t-s)\right) \nu_{\Omega}(s) \tilde{\theta}(s) d \sigma_{s} \quad \forall t \in \mathbb{R}^{n} \backslash \mathrm{cl} \Omega
$$

has a unique continuous extension to $\mathbb{R}^{n} \backslash \Omega$, which we still denote by $\tilde{u}$, and such an extension is the unique solution in $C_{\mathrm{loc}}^{m, \alpha}\left(\mathbb{R}^{n} \backslash \Omega\right)$ of the following problem

$$
\begin{cases}\Delta u(t)=0 & \forall t \in \mathbb{R}^{n} \backslash \mathrm{cl} \Omega \\ u(t)=g_{0}(t)-\int_{\partial \Omega} g_{0} \tau_{0} d \sigma & \forall t \in \partial \Omega \\ \lim _{t \rightarrow \infty} u(t)=0 & \end{cases}
$$

In [29], we have shown that the solutions of a periodic Dirichlet problem for the Laplace equation in $\mathbb{S}\left[\Omega_{p, \epsilon}\right]^{-}$ depend analytically upon $\epsilon$ and upon (a rescaling of) the Dirichlet datum. By Lemma 3.1 (ii), we know that (a rescaling of) the Dirichlet datum of the auxiliary problem (3.5) depends analytically upon $(\epsilon, g, f)$. Then we deduce that the solution of problem (3.5) depends analytically on $(\epsilon, g, f)$, and we have the following.

Proposition 3.3. Let $m \in \mathbb{N} \backslash\{0\}, \alpha \in] 0,1\left[\right.$. Let $\rho>0$. Let (3.1)-(3.4) hold. Then there exist $\left.\left.\epsilon_{1} \in\right] 0, \epsilon_{0}\right]$, an open neighborhood $\mathcal{U}$ of $g_{0}$ in $C^{m, \alpha}(\partial \Omega)$, an open neighborhood $\mathcal{V}$ of $f_{0}$ in $C_{q, \omega, \rho}^{0}\left(\mathbb{R}^{n}\right)_{0}$, and a real analytic $\operatorname{map}(\Theta[\cdot, \cdot, \cdot], \Xi[\cdot, \cdot, \cdot])$ from $]-\epsilon_{1}, \epsilon_{1}\left[\times \mathcal{U} \times \mathcal{V}\right.$ to $C^{m, \alpha}(\partial \Omega)_{0} \times \mathbb{R}$ such that

$$
u_{\#}[\epsilon, g, f]=w_{q}^{-}\left[\partial \Omega_{p, \epsilon}, \Theta[\epsilon, g, f]((\cdot-p) / \epsilon)\right]+\Xi[\epsilon, g, f] \quad \text { on } \operatorname{cl} \mathbb{S}\left[\Omega_{p, \epsilon}\right]^{-},
$$

for all $(\epsilon, g, f) \in] 0, \epsilon_{1}\left[\times \mathcal{U} \times \mathcal{V}\right.$. Moreover, $\left(\Theta\left[0, g_{0}, f_{0}\right], \Xi\left[0, g_{0}, f_{0}\right]\right)=(\tilde{\theta}, \tilde{\xi})$, where $\tilde{\theta}$, $\tilde{\xi}$ are as in Lemma 3.2.

Then we have the following representation Theorem for $u_{\#}[\cdot, \cdot, \cdot]$.

Theorem 3.4. Let $m \in \mathbb{N} \backslash\{0\}, \alpha \in] 0,1\left[\right.$. Let $\rho>0$. Let (3.1)-(3.4) hold. Let $\epsilon_{1}, \mathcal{U}, \mathcal{V}, \Xi$ be as in Proposition 3.3. Then the following statements hold.

(i) Let $V$ be a bounded open subset of $\mathbb{R}^{n}$ such that $\mathrm{cl} V \subseteq \mathbb{R}^{n} \backslash\left(p+q \mathbb{Z}^{n}\right)$. Let $r \in \mathbb{N}$. Then there exist $\left.\left.\epsilon_{\#, 2} \in\right] 0, \epsilon_{1}\right]$ and a real analytic map $U_{\#}$ from $]-\epsilon_{\#, 2}, \epsilon_{\#, 2}\left[\times \mathcal{U} \times \mathcal{V}\right.$ to $C^{r}(\mathrm{cl} V)$ such that the following statements hold.

(j) $\mathrm{cl} V \subseteq \mathbb{S}\left[\Omega_{p, \epsilon}\right]^{-}$for all $\left.\epsilon \in\right]-\epsilon_{\#, 2}, \epsilon_{\#, 2}[$.

(jj)

$$
u_{\#}[\epsilon, g, f](x)=\epsilon^{n-1} U_{\#}[\epsilon, g, f](x)+\Xi[\epsilon, g, f] \quad \forall x \in \operatorname{cl} V,
$$

for all $(\epsilon, g, f) \in] 0, \epsilon_{\#, 2}[\times \mathcal{U} \times \mathcal{V}$. Moreover,

$$
\begin{aligned}
U_{\#}\left[0, g_{0}, f_{0}\right](x)= & -\int_{\partial \Omega}\left(D S_{q, n}(x-p)\right) \nu_{\Omega}(s) \tilde{\theta}(s) d \sigma_{s} \\
= & D S_{q, n}(x-p) \int_{\partial \Omega} \nu_{\Omega}(s) \tilde{u}(s) d \sigma_{s} \\
& -D S_{q, n}(x-p) \int_{\partial \Omega} s \frac{\partial \tilde{u}}{\partial \nu_{\Omega}}(s) d \sigma_{s} \quad \forall x \in \mathrm{cl} V,
\end{aligned}
$$

where $\tilde{\theta}, \tilde{u}$ are as in Lemma 3.2. 
(ii) Let $\widetilde{V}$ be a bounded open subset of $\mathbb{R}^{n} \backslash \mathrm{cl} \Omega$. Then there exist $\left.\left.\tilde{\epsilon}_{\#, 2} \in\right] 0, \epsilon_{1}\right]$ and a real analytic map $\widetilde{U}_{\#}$ from $]-\tilde{\epsilon}_{\#, 2}, \tilde{\epsilon}_{\#, 2}\left[\times \mathcal{U} \times \mathcal{V}\right.$ to $C^{m, \alpha}(\mathrm{cl} \widetilde{V})$ such that the following statements hold.

(j') $p+\epsilon \mathrm{cl} \tilde{V} \subseteq Q \backslash \Omega_{p, \epsilon}$ for all $\left.\epsilon \in\right]-\tilde{\epsilon}_{\#, 2}, \tilde{\epsilon}_{\#, 2} \backslash \backslash\{0\}$.

$\left(j j^{\prime}\right)$

$$
u_{\#}[\epsilon, g, f](p+\epsilon t)=\widetilde{U}_{\#}[\epsilon, g, f](t)+\Xi[\epsilon, g, f] \quad \forall t \in \mathrm{cl} \widetilde{V},
$$

for all $(\epsilon, g, f) \in] 0, \tilde{\epsilon}_{\#, 2}[\times \mathcal{U} \times \mathcal{V}$. Moreover,

$$
\widetilde{U}_{\#}\left[0, g_{0}, f_{0}\right](t)=\tilde{u}(t) \quad \forall t \in \mathrm{cl} \widetilde{V} .
$$

where $\tilde{u}$ is as in Lemma 3.2.

Proof. We follow the argument of [29, §4]. We first consider statement (i). By taking $\left.\left.\epsilon_{\#, 2} \in\right] 0, \epsilon_{1}\right]$ small enough, we can clearly assume that (j) holds. Consider now (jj). By Proposition 3.3, if $(\epsilon, g, f) \in] 0, \epsilon_{\#, 2}[\times \mathcal{U} \times \mathcal{V}$, we have

$$
u_{\#}[\epsilon, g, f](x)=-\epsilon^{n-1} \int_{\partial \Omega}\left(D S_{q, n}(x-p-\epsilon s)\right) \nu_{\Omega}(s) \Theta[\epsilon, g, f](s) d \sigma_{s}+\Xi[\epsilon, g, f]
$$

$\forall x \in \mathrm{cl} V$.

Thus it is natural to set

$$
U_{\#}[\epsilon, g, f](x) \equiv-\int_{\partial \Omega}\left(D S_{q, n}(x-p-\epsilon s)\right) \nu_{\Omega}(s) \Theta[\epsilon, g, f](s) d \sigma_{s} \quad \forall x \in \mathrm{cl} V,
$$

for all $(\epsilon, g, f) \in]-\epsilon_{\#, 2}, \epsilon_{\#, 2}[\times \mathcal{U} \times \mathcal{V}$. Then Proposition 3.3, standard properties of integral operators with real analytic kernels and with no singularity (cf. e.g., [23, §4]), and classical potential theory (cf. e.g., Miranda [27], Lanza and Rossi [25, Thm. 3.1]) imply that $U_{\#}$ is a real analytic map from $]-\epsilon_{\#, 2}, \epsilon_{\#, 2}\left[\times \mathcal{U} \times \mathcal{V}\right.$ to $C^{r}(\operatorname{cl} V)$ such that (jj) holds (see also $[29, \S 4]$.

Consider now (ii). Let $R>0$ be such that $(\mathrm{cl} \widetilde{V} \cup \mathrm{cl} \Omega) \subseteq \mathbb{B}_{n}(0, R)$. By the continuity of the restriction operator from $C^{m, \alpha}\left(\mathrm{clB}_{n}(0, R) \backslash \Omega\right)$ to $C^{m, \alpha}(\mathrm{cl} \widetilde{V})$, it suffices to prove statement (ii) with $\widetilde{V}$ replaced by $\mathbb{B}_{n}(0, R) \backslash \operatorname{cl} \Omega$. By taking $\left.\left.\tilde{\epsilon}_{\#, 2} \in\right] 0, \epsilon_{1}\right]$ small enough, we can assume that

$$
\left.p+\epsilon \mathrm{clB}_{n}(0, R) \subseteq Q \quad \forall \epsilon \in\right]-\tilde{\epsilon}_{\#, 2}, \tilde{\epsilon}_{\#, 2}[.
$$

If $(\epsilon, g, f) \in] 0, \tilde{\epsilon}_{\#, 2}[\times \mathcal{U} \times \mathcal{V}$, a simple computation based on the Theorem of change of variables in integrals shows that

$$
\begin{aligned}
u_{\#}[\epsilon, g, f](p+\epsilon t)= & -\int_{\partial \Omega}\left(D S_{n}(t-s)\right) \nu_{\Omega}(s) \Theta[\epsilon, g, f](s) d \sigma_{s} \\
& -\epsilon^{n-1} \int_{\partial \Omega}\left(D R_{n}(\epsilon(t-s))\right) \nu_{\Omega}(s) \Theta[\epsilon, g, f](s) d \sigma_{s}+\Xi[\epsilon, g, f]
\end{aligned}
$$

for all $t \in \mathrm{clB}_{n}(0, R) \backslash \mathrm{cl} \Omega$. If $\left.(\epsilon, g, f) \in\right]-\tilde{\epsilon}_{\#, 2}, \tilde{\epsilon}_{\#, 2}[\times \mathcal{U} \times \mathcal{V}$, classical potential theory implies that the function

$$
-\int_{\partial \Omega}\left(D S_{n}(t-s)\right) \nu_{\Omega}(s) \Theta[\epsilon, g, f](s) d \sigma_{s}
$$

of the variable $t \in \mathrm{cl} \mathbb{B}_{n}(0, R) \backslash \mathrm{cl} \Omega$ admits an extension to $\operatorname{cl} \mathbb{B}_{n}(0, R) \backslash \Omega$ of class $C^{m, \alpha}\left(\mathrm{clB}_{n}(0, R) \backslash \Omega\right)$, which we denote by $w^{-}[\partial \Omega, \Theta[\epsilon, g, f]]_{\mid c \mathbb{B}_{n}(0, R) \backslash \Omega}$ (cf. e.g., Miranda [27], Lanza and Rossi [25, Thm. 3.1].) Then classical potential theory and Proposition 3.3 imply that the map from $]-\tilde{\epsilon}_{\#, 2}, \tilde{\epsilon}_{\#, 2}\left[\times \mathcal{U} \times \mathcal{V}\right.$ to $C^{m, \alpha}\left(\mathrm{clB}_{n}(0, R) \backslash \Omega\right)$ which takes $(\epsilon, g, f)$ to $w^{-}[\partial \Omega, \Theta[\epsilon, g, f]]_{|c| \mathbb{B}_{n}(0, R) \backslash \Omega}$ is real analytic (cf. e.g., Miranda [27], Lanza and Rossi [25, Thm. 3.1].) Therefore, if we set

$$
\begin{aligned}
\widetilde{U}_{\#}[\epsilon, g, f](t) & \equiv w^{-}[\partial \Omega, \Theta[\epsilon, g, f]]_{|c| \mathbb{B}_{n}(0, R) \backslash \Omega}(t) \\
& -\epsilon^{n-1} \int_{\partial \Omega}\left(D R_{n}(\epsilon(t-s))\right) \nu_{\Omega}(s) \Theta[\epsilon, g, f](s) d \sigma_{s} \quad \forall t \in \operatorname{clB}_{n}(0, R) \backslash \Omega,
\end{aligned}
$$

for all $(\epsilon, g, f) \in]-\tilde{\epsilon}_{\#, 2}, \tilde{\epsilon}_{\#, 2}[\times \mathcal{U} \times \mathcal{V}$, Proposition 3.3, standard properties of integral operators with real analytic kernels and with no singularity (cf. e.g., $[23, \S 4]$ ), and classical potential theory imply that $\widetilde{U}_{\#}$ is a real analytic map from $]-\tilde{\epsilon}_{\#, 2}, \tilde{\epsilon}_{\#, 2}\left[\times \mathcal{U} \times \mathcal{V}\right.$ to $C^{m, \alpha}\left(\operatorname{clB}_{n}(0, R) \backslash \Omega\right)$ such that (jj') holds with $\widetilde{V}$ replaced by $\mathbb{B}_{n}(0, R) \backslash \mathrm{cl} \Omega$ (see also $[29, \S 4]$.) Thus the proof is complete. 
Then we analyze the behaviour of the energy integral by means of the following.

Theorem 3.5. Let $m \in \mathbb{N} \backslash\{0\}, \alpha \in] 0,1\left[\right.$. Let (3.1)-(3.4) hold. Let $\epsilon_{1}, \mathcal{U}, \mathcal{V}$ be as in Proposition 3.3. Then there exist $\left.\left.\epsilon_{\#, 3} \in\right] 0, \epsilon_{1}\right]$ and a real analytic map $G_{\#}$ from $]-\epsilon_{\#, 3}, \epsilon_{\#, 3}[\times \mathcal{U} \times \mathcal{V}$ to $\mathbb{R}$, such that

$$
\int_{Q \backslash \mathrm{cl} \Omega_{p, \epsilon}}\left|D_{x} u_{\#}[\epsilon, g, f](x)\right|^{2} d x=\epsilon^{n-2} G_{\#}[\epsilon, g, f],
$$

for all $(\epsilon, g, f) \in] 0, \epsilon_{\#, 3}[\times \mathcal{U} \times \mathcal{V}$. Moreover,

$$
G_{\#}\left[0, g_{0}, f_{0}\right]=\int_{\mathbb{R}^{n} \backslash \mathrm{cl} \Omega}|D \tilde{u}(t)|^{2} d t,
$$

where $\tilde{u}$ is as in Lemma 3.2.

Proof. We follow the argument of $[29, \S 4]$. Let $(\epsilon, g, f) \in] 0, \epsilon_{1}[\times \mathcal{U} \times \mathcal{V}$. By the Green Formula and by the periodicity of $u_{\#}[\epsilon, g, f](\cdot)$, we have

$$
\begin{aligned}
\int_{Q \backslash \mathrm{cl} \Omega_{p, \epsilon}}\left|D_{x} u_{\#}[\epsilon, g, f](x)\right|^{2} d x \\
=-\epsilon^{n-1} \int_{\partial \Omega} D_{x} u_{\#}[\epsilon, g, f](p+\epsilon t) \nu_{\Omega}(t) u_{\#}[\epsilon, g, f](p+\epsilon t) d \sigma_{t} \\
=-\epsilon^{n-2} \int_{\partial \Omega} D\left(u_{\#}[\epsilon, g, f] \circ\left(p+\epsilon \operatorname{id}_{n}\right)\right)(t) \nu_{\Omega}(t)\left(g(t)-P_{q}[f](p+\epsilon t)\right) d \sigma_{t},
\end{aligned}
$$

where $\operatorname{id}_{n}$ denotes the identity in $\mathbb{R}^{n}$. Let $R>0$ be such that $\mathrm{cl} \Omega \subseteq \mathbb{B}_{n}(0, R)$. By Proposition 3.3 and Theorem 3.4 (ii), there exist $\left.\left.\epsilon_{\#, 3} \in\right] 0, \epsilon_{1}\right]$ and a real analytic map $\widehat{U}_{\#}$ from $]-\epsilon_{\#, 3}, \epsilon_{\#, 3}\left[\times \mathcal{U} \times \mathcal{V}\right.$ to $C^{m, \alpha}\left(\operatorname{cl} \mathbb{B}_{n}(0, R) \backslash \Omega\right)$, such that

and that

$$
\left.p+\epsilon \operatorname{cl}\left(\mathbb{B}_{n}(0, R) \backslash \operatorname{cl} \Omega\right) \subseteq Q \backslash \Omega_{p, \epsilon} \quad \forall \epsilon \in\right]-\epsilon_{\#, 3}, \epsilon_{\#, 3}\lfloor\backslash\{0\}
$$

$$
\widehat{U}_{\#}[\epsilon, g, f](t)=u_{\#}[\epsilon, g, f] \circ\left(p+\epsilon \mathrm{id}_{n}\right)(t) \quad \forall t \in \operatorname{clB}_{n}(0, R) \backslash \Omega,
$$

for all $(\epsilon, g, f) \in] 0, \epsilon_{\#, 3}[\times \mathcal{U} \times \mathcal{V}$, and that

$$
\widehat{U}_{\#}\left[0, g_{0}, f_{0}\right](t)=\tilde{u}(t)+\tilde{\xi} \quad \forall t \in \operatorname{cl} \mathbb{B}_{n}(0, R) \backslash \Omega,
$$

where $\tilde{u}, \tilde{\xi}$ are as in Lemma 3.2. By equality (3.7), we have

$$
\begin{aligned}
\int_{Q \backslash \mathrm{cl} \Omega_{p, \epsilon}} \mid & \left.D_{x} u_{\#}[\epsilon, g, f](x)\right|^{2} d x \\
= & -\epsilon^{n-2} \int_{\partial \Omega} D_{t} \widehat{U}_{\#}[\epsilon, g, f](t) \nu_{\Omega}(t)\left(g(t)-P_{q}[f](p+\epsilon t)\right) d \sigma_{t},
\end{aligned}
$$

for all $(\epsilon, g, f) \in] 0, \epsilon_{\#, 3}[\times \mathcal{U} \times \mathcal{V}$. Thus it is natural to set

$$
G_{\#}[\epsilon, g, f] \equiv-\int_{\partial \Omega} D_{t} \widehat{U}_{\#}[\epsilon, g, f](t) \nu_{\Omega}(t)\left(g(t)-P_{q}[f](p+\epsilon t)\right) d \sigma_{t},
$$

for all $(\epsilon, g, f) \in]-\epsilon_{\#, 3}, \epsilon_{\#, 3}\left[\times \mathcal{U} \times \mathcal{V}\right.$. Then by continuity of the partial derivatives from $C^{m, \alpha}\left(\mathrm{cl} \mathbb{B}_{n}(0, R) \backslash \Omega\right)$ to $C^{m-1, \alpha}\left(\operatorname{clB}_{n}(0, R) \backslash \Omega\right)$, and by continuity of the trace operator on $\partial \Omega$ from $C^{m-1, \alpha}\left(\mathrm{cl} \mathbb{B}_{n}(0, R) \backslash \Omega\right)$ to $C^{m-1, \alpha}(\partial \Omega)$, and by the continuity of the pointwise product in Schauder spaces, and by Lemma 3.1 (ii), and by classical potential theory, we conclude that $G_{\#}$ is a real analytic map from $]-\epsilon_{\#, 3}, \epsilon_{\#, 3}[\times \mathcal{U} \times \mathcal{V}$ to $\mathbb{R}$ and that the Theorem holds (see also [29, §4].)

Finally, we consider the integral of $u_{\#}[\cdot, \cdot, \cdot]$, and we prove the following.

Theorem 3.6. Let $m \in \mathbb{N} \backslash\{0\}, \alpha \in] 0,1\left[\right.$. Let (3.1)-(3.4) hold. Let $\epsilon_{1}, \mathcal{U}, \mathcal{V}$ be as in Proposition 3.3. Then there exists a real analytic map $J_{\#}$ from $]-\epsilon_{1}, \epsilon_{1}[\times \mathcal{U} \times \mathcal{V}$ to $\mathbb{R}$, such that

$$
\int_{Q \backslash \mathrm{cl} \Omega_{p, \epsilon}} u_{\#}[\epsilon, g, f](x) d x=J_{\#}[\epsilon, g, f],
$$

for all $(\epsilon, g, f) \in] 0, \epsilon_{1}[\times \mathcal{U} \times \mathcal{V}$. Moreover,

$$
J_{\#}\left[0, g_{0}, f_{0}\right]=\tilde{\xi} \operatorname{meas}(Q),
$$

where $\tilde{\xi}$ is as in Lemma 3.2. 
Proof. Let $(\epsilon, g, f) \in] 0, \epsilon_{1}[\times \mathcal{U} \times \mathcal{V}$. Clearly,

$$
\begin{aligned}
\int_{Q \backslash \mathrm{c} \Omega_{p, \epsilon}} u_{\#}[\epsilon, g, f](x) d x= & \int_{Q \backslash \mathrm{c} 1 \Omega_{p, \epsilon}} w_{q}^{-}\left[\partial \Omega_{p, \epsilon}, \Theta[\epsilon, g, f]((\cdot-p) / \epsilon)\right](x) d x \\
& +\Xi[\epsilon, g, f]\left(\operatorname{meas}(Q)-\epsilon^{n} \operatorname{meas}(\Omega)\right),
\end{aligned}
$$

where meas $(Q)$ and meas $(\Omega)$ denote the $n$-dimensional measure of $Q$ and of $\Omega$, respectively. By equality (2.2), we have

$$
\begin{aligned}
& w_{q}^{-} {\left[\partial \Omega_{p, \epsilon}, \Theta[\epsilon, g, f]((\cdot-p) / \epsilon)\right](x) } \\
&=-\sum_{j=1}^{n} \frac{\partial}{\partial x_{j}} v_{q}^{-}\left[\partial \Omega_{p, \epsilon}, \Theta[\epsilon, g, f]((\cdot-p) / \epsilon)\left(\nu_{\Omega_{p, \epsilon}}(\cdot)\right)_{j}\right](x) \quad \forall x \in \operatorname{cl} Q \backslash \mathrm{cl} \Omega_{p, \epsilon} .
\end{aligned}
$$

Let $j \in\{1, \ldots, n\}$. By the Divergence Theorem and the periodicity of the periodic simple layer potential, we have

$$
\begin{aligned}
\int_{Q \backslash \mathrm{c} \backslash \Omega_{p, \epsilon}} & \frac{\partial}{\partial x_{j}} v_{q}^{-}\left[\partial \Omega_{p, \epsilon}, \Theta[\epsilon, g, f]((\cdot-p) / \epsilon)\left(\nu_{\Omega_{p, \epsilon}}(\cdot)\right)_{j}\right](x) d x \\
= & \int_{\partial Q} v_{q}^{-}\left[\partial \Omega_{p, \epsilon}, \Theta[\epsilon, g, f]((\cdot-p) / \epsilon)\left(\nu_{\Omega_{p, \epsilon}}(\cdot)\right)_{j}\right](x)\left(\nu_{Q}(x)\right)_{j} d \sigma_{x} \\
& -\int_{\partial \Omega_{p, \epsilon}} v_{q}^{-}\left[\partial \Omega_{p, \epsilon}, \Theta[\epsilon, g, f]((\cdot-p) / \epsilon)\left(\nu_{\Omega_{p, \epsilon}}(\cdot)\right)_{j}\right](x)\left(\nu_{\Omega_{p, \epsilon}}(x)\right)_{j} d \sigma_{x} \\
= & -\epsilon^{n-1} \int_{\partial \Omega} v_{q}^{-}\left[\partial \Omega_{p, \epsilon}, \Theta[\epsilon, g, f]((\cdot-p) / \epsilon)\left(\nu_{\Omega_{p, \epsilon}}(\cdot)\right)_{j}\right](p+\epsilon t)\left(\nu_{\Omega}(t)\right)_{j} d \sigma_{t} .
\end{aligned}
$$

Then we note that

$$
\begin{aligned}
v_{q}^{-}\left[\partial \Omega_{p, \epsilon}, \Theta[\epsilon, g, f]((\cdot-p) / \epsilon)\left(\nu_{\Omega_{p, \epsilon}}(\cdot)\right)_{j}\right](p+\epsilon t) & \\
= & \epsilon^{n-1} \int_{\partial \Omega} S_{n}(\epsilon(t-s)) \Theta[\epsilon, g, f](s)\left(\nu_{\Omega}(s)\right)_{j} d \sigma_{s} \\
& +\epsilon^{n-1} \int_{\partial \Omega} R_{n}(\epsilon(t-s)) \Theta[\epsilon, g, f](s)\left(\nu_{\Omega}(s)\right)_{j} d \sigma_{s} \quad \forall t \in \partial \Omega .
\end{aligned}
$$

We now observe that if $\epsilon>0$ and $x \in \mathbb{R}^{n} \backslash\{0\}$ then we have

$$
S_{n}(\epsilon x)=\epsilon^{2-n} S_{n}(x)+\delta_{2, n} \frac{1}{2 \pi} \log \epsilon .
$$

Moreover, by the Divergence Theorem, it's immediate to see that

$$
\begin{aligned}
\int_{\partial \Omega}\left(\int_{\partial \Omega} \Theta[\epsilon, g, f](s)\left(\nu_{\Omega}(s)\right)_{j} d \sigma_{s}\right)\left(\nu_{\Omega}(t)\right)_{j} d \sigma_{t} \\
=\left(\int_{\partial \Omega} \Theta[\epsilon, g, f](s)\left(\nu_{\Omega}(s)\right)_{j} d \sigma_{s}\right)\left(\int_{\partial \Omega}\left(\nu_{\Omega}(t)\right)_{j} d \sigma_{t}\right)=0 .
\end{aligned}
$$

Hence, by equalities (3.10) and (3.11), if $(\epsilon, g, f) \in] 0, \epsilon_{1}[\times \mathcal{U} \times \mathcal{V}$, we have

$$
\begin{aligned}
\int_{Q \backslash \mathrm{c} \backslash \Omega_{p, \epsilon}} w_{q}^{-} & {\left[\partial \Omega_{p, \epsilon}, \Theta[\epsilon, g, f]((\cdot-p) / \epsilon)\right](x) d x } \\
= & \sum_{j=1}^{n} \epsilon^{n}\left[\int_{\partial \Omega}\left(\int_{\partial \Omega} S_{n}(t-s) \Theta[\epsilon, g, f](s)\left(\nu_{\Omega}(s)\right)_{j} d \sigma_{s}\right)\left(\nu_{\Omega}(t)\right)_{j} d \sigma_{t}\right. \\
& \left.+\epsilon^{n-2} \int_{\partial \Omega}\left(\int_{\partial \Omega} R_{n}(\epsilon(t-s)) \Theta[\epsilon, g, f](s)\left(\nu_{\Omega}(s)\right)_{j} d \sigma_{s}\right)\left(\nu_{\Omega}(t)\right)_{j} d \sigma_{t}\right] .
\end{aligned}
$$

Thus we set

$$
\begin{aligned}
\tilde{J}_{\#}[\epsilon, g, f] & \equiv \sum_{j=1}^{n}\left[\int_{\partial \Omega}\left(\int_{\partial \Omega} S_{n}(t-s) \Theta[\epsilon, g, f](s)\left(\nu_{\Omega}(s)\right)_{j} d \sigma_{s}\right)\left(\nu_{\Omega}(t)\right)_{j} d \sigma_{t}\right. \\
& \left.+\epsilon^{n-2} \int_{\partial \Omega}\left(\int_{\partial \Omega} R_{n}(\epsilon(t-s)) \Theta[\epsilon, g, f](s)\left(\nu_{\Omega}(s)\right)_{j} d \sigma_{s}\right)\left(\nu_{\Omega}(t)\right)_{j} d \sigma_{t}\right]
\end{aligned}
$$


for all $(\epsilon, g, f) \in]-\epsilon_{1}, \epsilon_{1}[\times \mathcal{U} \times \mathcal{V}$. Clearly, if $(\epsilon, g, f) \in] 0, \epsilon_{1}[\times \mathcal{U} \times \mathcal{V}$, then

$$
\int_{Q \backslash \mathrm{c} 1 \Omega_{p, \epsilon}} w_{q}^{-}\left[\partial \Omega_{p, \epsilon}, \Theta[\epsilon, g, f]((\cdot-p) / \epsilon)\right](x) d x=\epsilon^{n} \tilde{J}_{\#}[\epsilon, g, f] .
$$

Then the analyticity of $\Theta$, the continuity of the linear map from $C^{m-1, \alpha}(\partial \Omega)$ to $C^{m, \alpha}(\partial \Omega)$ which takes $f$ to the function $\int_{\partial \Omega} S_{n}(t-s) f(s) d \sigma_{s}$ of the variable $t \in \partial \Omega$ (cf. e.g., Miranda [27], Lanza and Rossi [25, Thm. 3.1]), the continuity of the pointwise product in Schauder spaces, standard properties of integral operators with real analytic kernels and with no singularity (cf. e.g., $[23, \S 4]$ ), and standard calculus in Banach spaces imply that the map $\tilde{J}_{\#}$ is real analytic from $]-\epsilon_{1}, \epsilon_{1}[\times \mathcal{U} \times \mathcal{V}$ to $\mathbb{R}$. Hence, if we set

$$
J_{\#}[\epsilon, g, f] \equiv \epsilon^{n} \tilde{J}_{\#}[\epsilon, g, f]+\Xi[\epsilon, g, f]\left(\operatorname{meas}(Q)-\epsilon^{n} \operatorname{meas}(\Omega)\right)
$$

for all $(\epsilon, g, f) \in]-\epsilon_{1}, \epsilon_{1}\left[\times \mathcal{U} \times \mathcal{V}\right.$, we immediately deduce that $J_{\#}$ is a real analytic map from $]-\epsilon_{1}, \epsilon_{1}[\times \mathcal{U} \times \mathcal{V}$ to $\mathbb{R}$ such that equalities (3.8), (3.9) hold, and thus the proof is complete.

\section{A functional analytic representation Theorem for the solution of problem (1.1)}

In this Section, we deduce by the results of Section 3 for $u_{\#}[\cdot, \cdot, \cdot]$ the corresponding results for $u[\cdot, \cdot, \cdot]$. By formula (3.6) and by Theorem 3.4, we immediately deduce the following.

Theorem 4.1. Let $m \in \mathbb{N} \backslash\{0\}, \alpha \in] 0,1\left[\right.$. Let $\rho>0$. Let (3.1)-(3.4) hold. Let $\epsilon_{1}, \mathcal{U}, \mathcal{V}$ be as in Proposition 3.3. Then the following statements hold.

(i) Let $V$ be a bounded open subset of $\mathbb{R}^{n}$ such that $\operatorname{cl} V \subseteq \mathbb{R}^{n} \backslash\left(p+q \mathbb{Z}^{n}\right)$. Let $r \in \mathbb{N}$. Then there exist $\left.\left.\epsilon_{2} \in\right] 0, \epsilon_{1}\right]$ and a real analytic map $U$ from $]-\epsilon_{2}, \epsilon_{2}\left[\times \mathcal{U} \times \mathcal{V}\right.$ to $C^{r}(\mathrm{cl} V)$ such that the following statements hold.

(j) $\operatorname{cl} V \subseteq \mathbb{S}\left[\Omega_{p, \epsilon}\right]^{-}$for all $\left.\epsilon \in\right]-\epsilon_{2}, \epsilon_{2}[$.

$(j j)$

$$
u[\epsilon, g, f](x)=U[\epsilon, g, f](x)+P_{q}[f](x) \quad \forall x \in \mathrm{cl} V,
$$

for all $(\epsilon, g, f) \in] 0, \epsilon_{2}[\times \mathcal{U} \times \mathcal{V}$. Moreover,

$$
U\left[0, g_{0}, f_{0}\right](x)=\tilde{\xi} \quad \forall x \in \operatorname{cl} V,
$$

where $\tilde{\xi}$ is as in Lemma 3.2.

(ii) Let $\widetilde{V}$ be a bounded open subset of $\mathbb{R}^{n} \backslash \mathrm{cl} \Omega$. Then there exist $\left.\left.\tilde{\epsilon}_{2} \in\right] 0, \epsilon_{1}\right]$ and a real analytic map $\widetilde{U}$ from ]$-\tilde{\epsilon}_{2}, \tilde{\epsilon}_{2}\left[\times \mathcal{U} \times \mathcal{V}\right.$ to $C^{m, \alpha}(\mathrm{cl} \widetilde{V})$ such that the following statements hold.

$\left(j^{\prime}\right) p+\epsilon \mathrm{cl} \widetilde{V} \subseteq Q \backslash \Omega_{p, \epsilon}$ for all $\left.\epsilon \in\right]-\tilde{\epsilon}_{2}, \tilde{\epsilon}_{2}\lfloor\backslash\{0\}$.

$\left(j j^{\prime}\right)$

$$
u[\epsilon, g, f](p+\epsilon t)=\widetilde{U}[\epsilon, g, f](t)+P_{q}[f](p+\epsilon t) \quad \forall t \in \mathrm{cl} \widetilde{V},
$$

for all $(\epsilon, g, f) \in] 0, \tilde{\epsilon}_{2}[\times \mathcal{U} \times \mathcal{V}$. Moreover,

$$
\widetilde{U}\left[0, g_{0}, f_{0}\right](t)=\tilde{u}(t)+\tilde{\xi} \quad \forall t \in \mathrm{cl} \widetilde{V} .
$$

where $\tilde{u}, \tilde{\xi}$ are as in Lemma 3.2.

As far as the energy integral of the solution is concerned, we have the following.

Theorem 4.2. Let $m \in \mathbb{N} \backslash\{0\}, \alpha \in] 0,1\left[\right.$. Let (3.1)-(3.4) hold. Let $\epsilon_{1}, \mathcal{U}, \mathcal{V}$ be as in Proposition 3.3. Then there exist $\left.\left.\epsilon_{3} \in\right] 0, \epsilon_{1}\right]$ and a real analytic map $G$ from $]-\epsilon_{3}, \epsilon_{3}[\times \mathcal{U} \times \mathcal{V}$ to $\mathbb{R}$, such that

$$
\int_{Q \backslash \mathrm{c} \backslash \Omega_{p, \epsilon}}\left|D_{x} u[\epsilon, g, f](x)\right|^{2} d x=\epsilon^{n-2} G[\epsilon, g, f]+\int_{Q}\left|D_{x} P_{q}[f](x)\right|^{2} d x,
$$

for all $(\epsilon, g, f) \in] 0, \epsilon_{3}[\times \mathcal{U} \times \mathcal{V}$. Moreover,

$$
G\left[0, g_{0}, f_{0}\right]=\int_{\mathbb{R}^{n} \backslash \mathrm{cl} \Omega}|D \tilde{u}(t)|^{2} d t,
$$

where $\tilde{u}$ is as in Lemma 3.2. 
Proof. Let $\epsilon_{\#, 3}, G_{\#}$ be as in Theorem 3.5. If $\left.(\epsilon, g, f) \in\right] 0, \epsilon_{\#, 3}[\times \mathcal{U} \times \mathcal{V}$, then we have

$$
\begin{aligned}
& \int_{Q \backslash \mathrm{cl} \Omega_{p, \epsilon}}\left|D_{x} u[\epsilon, g, f](x)\right|^{2} d x=\int_{Q \backslash \mathrm{cl} \Omega_{p, \epsilon}}\left|D_{x} u_{\#}[\epsilon, g, f](x)\right|^{2} d x \\
& +2 \int_{Q \backslash \mathrm{cl} \Omega_{p, \epsilon}} D_{x} u_{\#}[\epsilon, g, f](x) \cdot D_{x} P_{q}[f](x) d x+\int_{Q \backslash \mathrm{cl} \Omega_{p, \epsilon}}\left|D_{x} P_{q}[f](x)\right|^{2} d x .
\end{aligned}
$$

By the Divergence Theorem, by the harmonicity of $u_{\#}[\epsilon, g, f]$, and by the periodicity of $u_{\#}[\epsilon, g, f]$ and $P_{q}[f]$, we have

$$
\begin{aligned}
2 \int_{Q \backslash \mathrm{cl} \Omega_{p, \epsilon}} D_{x} & u_{\#}[\epsilon, g, f](x) \cdot D_{x} P_{q}[f](x) d x \\
& =-2 \epsilon^{n-1} \int_{\partial \Omega} P_{q}[f](p+\epsilon t)\left(\frac{\partial u_{\#}[\epsilon, g, f]}{\partial \nu_{\Omega_{p, \epsilon}}}\right)(p+\epsilon t) d \sigma_{t} \\
& =-2 \epsilon^{n-2} \int_{\partial \Omega} P_{q}[f](p+\epsilon t) D\left(u_{\#}[\epsilon, g, f] \circ\left(p+\epsilon \operatorname{id}_{n}\right)\right)(t) \nu_{\Omega}(t) d \sigma_{t},
\end{aligned}
$$

where $\mathrm{id}_{n}$ denotes the identity map in $\mathbb{R}^{n}$. Let $R>0$ be such that $\mathrm{cl} \Omega \subseteq \mathbb{B}_{n}(0, R)$. By Proposition 3.3 and Theorem 3.4 (ii), there exist $\left.\left.\epsilon_{3} \in\right] 0, \epsilon_{\#, 3}\right]$ and a real analytic map $\widehat{U}_{\#}$ from $]-\epsilon_{3}, \epsilon_{3}\left[\times \mathcal{U} \times \mathcal{V}\right.$ to $C^{m, \alpha}\left(\mathrm{clB} \mathbb{B}_{n}(0, R) \backslash\right.$ $\Omega)$, such that

$$
\left.p+\epsilon \operatorname{cl}\left(\mathbb{B}_{n}(0, R) \backslash \operatorname{cl} \Omega\right) \subseteq Q \backslash \Omega_{p, \epsilon} \quad \forall \epsilon \in\right]-\epsilon_{3}, \epsilon_{3}[\backslash\{0\},
$$

and that

$$
\widehat{U}_{\#}[\epsilon, g, f](t)=u_{\#}[\epsilon, g, f] \circ\left(p+\epsilon \operatorname{id}_{n}\right)(t) \quad \forall t \in \operatorname{clB}_{n}(0, R) \backslash \Omega,
$$

for all $(\epsilon, g, f) \in] 0, \epsilon_{3}[\times \mathcal{U} \times \mathcal{V}$, and that

$$
\widehat{U}_{\#}\left[0, g_{0}, f_{0}\right](t)=\tilde{u}(t)+\tilde{\xi} \quad \forall t \in \operatorname{clB}_{n}(0, R) \backslash \Omega,
$$

where $\tilde{u}, \tilde{\xi}$ are as in Lemma 3.2. Then we have

$$
\begin{aligned}
2 \int_{Q \backslash \mathrm{cl} \Omega_{p, \epsilon}} D_{x} u_{\#}[\epsilon, g, f](x) \cdot D_{x} P_{q}[f](x) d x \\
=-2 \epsilon^{n-2} \int_{\partial \Omega} P_{q}[f](p+\epsilon t) D_{t} \widehat{U}_{\#}[\epsilon, g, f](t) \nu_{\Omega}(t) d \sigma_{t} .
\end{aligned}
$$

for all $(\epsilon, g, f) \in] 0, \epsilon_{3}[\times \mathcal{U} \times \mathcal{V}$. Thus it is natural to set

$$
G_{1}[\epsilon, g, f] \equiv-2 \int_{\partial \Omega} P_{q}[f](p+\epsilon t) D_{t} \widehat{U}_{\#}[\epsilon, g, f](t) \nu_{\Omega}(t) d \sigma_{t},
$$

for all $(\epsilon, g, f) \in]-\epsilon_{3}, \epsilon_{3}\left[\times \mathcal{U} \times \mathcal{V}\right.$. Then by continuity of the partial derivatives from $C^{m, \alpha}\left(\operatorname{cl} \mathbb{B}_{n}(0, R) \backslash \Omega\right)$ to $C^{m-1, \alpha}\left(\operatorname{clB}_{n}(0, R) \backslash \Omega\right)$, and by continuity of the trace operator on $\partial \Omega$ from $C^{m-1, \alpha}\left(\operatorname{clB} \mathbb{B}_{n}(0, R) \backslash \Omega\right)$ to $C^{m-1, \alpha}(\partial \Omega)$, and by the continuity of the pointwise product in Schauder spaces, and by Lemma 3.1 (ii), we conclude that $G_{1}[\cdot, \cdot, \cdot]$ is a real analytic map from $]-\epsilon_{3}, \epsilon_{3}[\times \mathcal{U} \times \mathcal{V}$ to $\mathbb{R}$. Moreover, by classical potential theory, we have

$$
G_{1}\left[0, g_{0}, f_{0}\right]=-2 \int_{\partial \Omega} P_{q}\left[f_{0}\right](p) \frac{\partial \tilde{u}}{\partial \nu_{\Omega}}(t) d \sigma_{t}=0
$$

(see also $[29, \S 4]$.) If $(\epsilon, f) \in] 0, \epsilon_{0}[\times \mathcal{V}$, then clearly

$$
\int_{Q \backslash \mathrm{cl} \Omega_{p, \epsilon}}\left|D_{x} P_{q}[f](x)\right|^{2} d x=\int_{Q}\left|D_{x} P_{q}[f](x)\right|^{2} d x-\epsilon^{n} \int_{\Omega}\left|D_{x} P_{q}[f](p+\epsilon t)\right|^{2} d t .
$$

By standard properties of functions in the Roumieu class, there exists $\left.\left.\rho^{\prime} \in\right] 0, \rho\right]$ such that the map from $C_{q, \omega, \rho}^{0}\left(\mathbb{R}^{n}\right)$ to $C_{q, \omega, \rho^{\prime}}^{0}\left(\mathbb{R}^{n}\right)$ which takes $f$ to $\left|D_{x} P_{q}[f](\cdot)\right|^{2}$ is real analytic (cf. e.g., the proof of Lanza [18, Prop. 2.25].) By arguing as in the proof of Lemma 3.1, one can show that the map from $]-\epsilon_{0}, \epsilon_{0}[\times \mathcal{V}$ to $C^{m, \alpha}(\operatorname{cl} \Omega)$ which takes $(\epsilon, f)$ to the function $\left|D_{x} P_{q}[f](p+\epsilon t)\right|^{2}$ of the variable $t \in \mathrm{cl} \Omega$ is real analytic. Then, by the continuity of the linear operator from $C^{m, \alpha}(\operatorname{cl} \Omega)$ to $\mathbb{R}$ which takes $h$ to $\int_{\Omega} h(y) d y$, we immediately deduce that the map from $]-\epsilon_{0}, \epsilon_{0}\left[\times \mathcal{V}\right.$ to $\mathbb{R}$ which takes $(\epsilon, f)$ to $\int_{\Omega}\left|D_{x} P_{q}[f](p+\epsilon t)\right|^{2} d t$ is real analytic. Thus if we set

$$
G[\epsilon, g, f] \equiv G_{\#}[\epsilon, g, f]+G_{1}[\epsilon, g, f]-\epsilon^{2} \int_{\Omega}\left|D_{x} P_{q}[f](p+\epsilon t)\right|^{2} d t
$$

for all $(\epsilon, g, f) \in]-\epsilon_{3}, \epsilon_{3}[\times \mathcal{U} \times \mathcal{V}$, we deduce that $G$ is a real analytic map from $]-\epsilon_{3}, \epsilon_{3}[\times \mathcal{U} \times \mathcal{V}$ to $\mathbb{R}$ such that equalities (4.1), (4.2) hold. 
Remark 4.3. We note that the map from $C_{q, \omega, \rho}^{0}\left(\mathbb{R}^{n}\right)$ to $\mathbb{R}$ which takes $f$ to $\int_{Q}\left|D_{x} P_{q}[f](x)\right|^{2} d x$ is real analytic.

Finally, we consider the integral of $u[\cdot, \cdot, \cdot]$ and we prove the following.

Theorem 4.4. Let $m \in \mathbb{N} \backslash\{0\}, \alpha \in] 0,1\left[\right.$. Let (3.1)-(3.4) hold. Let $\epsilon_{1}, \mathcal{U}, \mathcal{V}$ be as in Proposition 3.3. Then there exists a real analytic map $J$ from $]-\epsilon_{1}, \epsilon_{1}[\times \mathcal{U} \times \mathcal{V}$ to $\mathbb{R}$, such that

$$
\int_{Q \backslash \mathrm{c} 1 \Omega_{p, \epsilon}} u[\epsilon, g, f](x) d x=J[\epsilon, g, f]+\int_{Q} P_{q}[f](x) d x,
$$

for all $(\epsilon, g, f) \in] 0, \epsilon_{1}[\times \mathcal{U} \times \mathcal{V}$. Moreover,

$$
J\left[0, g_{0}, f_{0}\right]=\tilde{\xi} \operatorname{meas}(Q),
$$

where $\tilde{\xi}$ is as in Lemma 3.2.

Proof. Let $J_{\#}$ be as in Theorem 3.6. If we set

$$
J[\epsilon, g, f] \equiv J_{\#}[\epsilon, g, f]-\epsilon^{n} \int_{\Omega} P_{q}[f](p+\epsilon t) d t
$$

for all $(\epsilon, g, f) \in]-\epsilon_{1}, \epsilon_{1}[\times \mathcal{U} \times \mathcal{V}$, then clearly

$$
\int_{Q \backslash \mathrm{c} 1 \Omega_{p, \epsilon}} u[\epsilon, g, f](x) d x=J[\epsilon, g, f]+\int_{Q} P_{q}[f](x) d x,
$$

for all $(\epsilon, g, f) \in] 0, \epsilon_{1}\left[\times \mathcal{U} \times \mathcal{V}\right.$. By Lemma 3.1 (i) and by the continuity of the linear operator from $C^{m, \alpha}(\operatorname{cl} \Omega)$ to $\mathbb{R}$ which takes $h$ to $\int_{\Omega} h(y) d y$, we immediately deduce that $J$ is a real analytic map from $]-\epsilon_{1}, \epsilon_{1}[\times \mathcal{U} \times \mathcal{V}$ to $\mathbb{R}$ and that (4.3) holds.

Remark 4.5. We note that the map from $C_{q, \omega, \rho}^{0}\left(\mathbb{R}^{n}\right)$ to $\mathbb{R}$ which takes $f$ to $\int_{Q} P_{q}[f](x) d x$ is linear and continuous.

Remark 4.6. Let the assumptions of Proposition 3.3 hold. Let $\tilde{u}, \tilde{\xi}$ be as in Lemma 3.2. We observe that if $V$ is a bounded open subset of $\mathbb{R}^{n}$ such that $\operatorname{cl} V \subseteq \mathbb{R}^{n} \backslash\left(p+q \mathbb{Z}^{n}\right)$ and if $r \in \mathbb{N}$, then Theorem 4.1 (i) implies that

$$
\lim _{(\epsilon, g, f) \rightarrow\left(0, g_{0}, f_{0}\right)} u[\epsilon, g, f]=\tilde{\xi}+P_{q}\left[f_{0}\right] \quad \text { in } C^{r}(\mathrm{cl} V),
$$

for all $n \in \mathbb{N} \backslash\{0,1\}$. Similarly, by Theorem 4.4, we have

$$
\lim _{(\epsilon, g, f) \rightarrow\left(0, g_{0}, f_{0}\right)} \int_{Q \backslash \mathrm{c} \backslash \Omega_{p, \epsilon}} u[\epsilon, g, f](x) d x=\tilde{\xi} \operatorname{meas}(Q)+\int_{Q} P_{q}\left[f_{0}\right](x) d x,
$$

for all $n \in \mathbb{N} \backslash\{0,1\}$. Instead, Theorem 4.2 implies that

$$
\lim _{(\epsilon, g, f) \rightarrow\left(0, g_{0}, f_{0}\right)} \int_{Q \backslash \mathrm{cl} \Omega_{p, \epsilon}}\left|D_{x} u[\epsilon, g, f](x)\right|^{2} d x=\int_{Q}\left|D_{x} P_{q}\left[f_{0}\right](x)\right|^{2} d x,
$$

if $n \in \mathbb{N} \backslash\{0,1,2\}$, whereas

$$
\begin{aligned}
\lim _{(\epsilon, g, f) \rightarrow\left(0, g_{0}, f_{0}\right)} \int_{Q \backslash \mathrm{c} 1 \Omega_{p, \epsilon}} & \left|D_{x} u[\epsilon, g, f](x)\right|^{2} d x \\
& =\int_{\mathbb{R}^{n} \backslash \mathrm{c} \mid \Omega}|D \tilde{u}(t)|^{2} d t+\int_{Q}\left|D_{x} P_{q}\left[f_{0}\right](x)\right|^{2} d x,
\end{aligned}
$$

if $n=2$.

\section{A A real analyticity result for a composition operator}

In this Appendix, we introduce a slight variant of Preciso [31, Prop. 4.2.16, p. 51], Preciso [32, Prop. 1.1, p. 101] on the real analyticity of a composition operator. See also Lanza [15, Prop. 2.17, Rem. 2.19] and the slight variant of the argument of Preciso of the proof of Lanza [19, Prop. 9, p. 214].

Proposition A.1. Let $m, h, k \in \mathbb{N}, h, k \geq 1$. Let $\alpha \in] 0,1], \rho>0$. Let $\Omega, \Omega^{\prime}$ be bounded open connected subsets of $\mathbb{R}^{h}, \mathbb{R}^{k}$, respectively. Let $\Omega^{\prime}$ be of class $C^{1}$. Then the operator $T$ defined by

$$
T[u, v] \equiv u \circ v
$$

for all $(u, v) \in C_{\omega, \rho}^{0}(\operatorname{cl} \Omega) \times C^{m, \alpha}\left(\operatorname{cl} \Omega^{\prime}, \Omega\right)$ is real analytic from the open subset $C_{\omega, \rho}^{0}(\operatorname{cl} \Omega) \times C^{m, \alpha}\left(\operatorname{cl} \Omega^{\prime}, \Omega\right)$ of $C_{\omega, \rho}^{0}(\mathrm{cl} \Omega) \times C^{m, \alpha}\left(\mathrm{cl} \Omega^{\prime}, \mathbb{R}^{h}\right)$ to $C^{m, \alpha}\left(\mathrm{cl} \Omega^{\prime}\right)$. 


\section{Acknowledgment}

The author is endebted to Prof. M. Lanza de Cristoforis for proposing him the problem and for a number of comments which have improved the quality of the paper.

\section{References}

[1] H. Ammari and H. Kang, Polarization and moment tensors, volume 162 of Applied Mathematical Sciences. Springer, New York, 2007.

[2] L. P. Castro and E. Pesetskaya, A transmission problem with imperfect contact for an unbounded multiply connected domain. Math. Methods Appl. Sci. 33 (2010), 517-526.

[3] L. P. Castro, E. Pesetskaya, and S. V. Rogosin, Effective conductivity of a composite material with nonideal contact conditions. Complex Var. Elliptic Equ. 54 (2009), 1085-1100.

[4] D. Cioranescu and F. Murat, Un terme étrange venu d'ailleurs. In Nonlinear partial differential equations and their applications. Collège de France Seminar, Vol. II (Paris, 1979/1980), volume 60 of Res. Notes in Math., pages 98-138, 389-390. Pitman, Boston, Mass., 1982.

[5] D. Cioranescu and F. Murat, Un terme étrange venu d'ailleurs. II. In Nonlinear partial differential equations and their applications. Collège de France Seminar, Vol. III (Paris, 1980/1981), volume 70 of Res. Notes in Math., pages 154-178, 425-426. Pitman, Boston, Mass., 1982.

[6] M. Dalla Riva, Stokes flow in a singularly perturbed exterior domain. Complex Var. Elliptic Equ., (to appear).

[7] M. Dalla Riva and M. Lanza de Cristoforis, Microscopically weakly singularly perturbed loads for a nonlinear traction boundary value problem: a functional analytic approach. Complex Var. Elliptic Equ. 55 (2010), 771-794.

[8] M. Dalla Riva and M. Lanza de Cristoforis, A singularly perturbed nonlinear traction boundary value problem for linearized elastostatics. A functional analytic approach. Analysis (Munich) 30 (2010), 67-92.

[9] M. Dalla Riva, M. Lanza de Cristoforis, and P. Musolino, Volume potentials associated to general second order differential operators with constant coefficients, (in preparation).

[10] P. Drygas and V. Mityushev, Effective conductivity of unidirectional cylinders with interfacial resistance, Quart. J. Mech. Appl. Math. 62 (2009), 235-262.

[11] D. Gilbarg and N. S. Trudinger, Elliptic partial differential equations of second order, volume 224 of Grundlehren der Mathematischen Wissenschaften [Fundamental Principles of Mathematical Sciences]. Springer-Verlag, Berlin, second edition, 1983.

[12] Eh. I. Grigolyuk and L. A. Fil'shtinskij, Periodic piecewise homogeneous elastic structures, (in Russian), Nauka, Moscow, 1992.

[13] V. Kozlov, V. Maz'ya, and A. Movchan, Asymptotic analysis of fields in multi-structures. Oxford Mathematical Monographs. The Clarendon Press Oxford University Press, New York, 1999.

[14] M. Lanza de Cristoforis, Properties and pathologies of the composition and inversion operators in Schauder spaces. Rend. Accad. Naz. Sci. XL Mem. Mat. 15 (1991), 93-109.

[15] M. Lanza de Cristoforis, Differentiability properties of a composition operator. Rend. Circ. Mat. Palermo (2) Suppl. 56 (1998), 157-165.

[16] M. Lanza de Cristoforis, Asymptotic behaviour of the conformal representation of a Jordan domain with a small hole in Schauder spaces. Comput. Methods Funct. Theory 2 (2002), 1-27.

[17] M. Lanza de Cristoforis, Asymptotic behaviour of the conformal representation of a Jordan domain with a small hole, and relative capacity. In Complex analysis and dynamical systems, Proc. Conf. Karmiel, June 19-22, 2001, volume 364 of Contemp. Math., pages 155-167. Amer. Math. Soc., Providence, RI, 2004.

[18] M. Lanza de Cristoforis, A domain perturbation problem for the Poisson equation. Complex Var. Theory Appl. 50 (2005), 851-867. 
[19] M. Lanza de Cristoforis, Perturbation problems in potential theory, a functional analytic approach. J. Appl. Funct. Anal. 2 (2007), 197-222.

[20] M. Lanza de Cristoforis, Asymptotic behavior of the solutions of the Dirichlet problem for the Laplace operator in a domain with a small hole. A functional analytic approach. Analysis (Munich) 28 (2008), 63-93.

[21] M. Lanza de Cristoforis, A singular domain perturbation problem for the Poisson equation. In More progresses in analysis, Proceedings of the 5th international ISAAC congress, Catania, Italy, July 25-30, 2005, pages 955-965. World Scientific, Hackensack, NJ, 2009.

[22] M. Lanza de Cristoforis and P. Musolino, A perturbation result for periodic layer potentials of general second order differential operators with constant coefficients. Far East J. Math. Sci. (FJMS) 52 (2011), $75-120$.

[23] M. Lanza de Cristoforis and P. Musolino, A real analyticity result for a nonlinear integral operator. Submitted, 2011.

[24] M. Lanza de Cristoforis and P. Musolino, A singularly perturbed nonlinear Robin problem in a periodically perforated domain: a functional analytic approach. Complex Var. Elliptic Equ., (to appear).

[25] M. Lanza de Cristoforis and L. Rossi, Real analytic dependence of simple and double layer potentials upon perturbation of the support and of the density. J. Integral Equations Appl. 16 (2004), 137-174.

[26] V. Maz'ya, S. Nazarov, and B. Plamenevskij, Asymptotic theory of elliptic boundary value problems in singularly perturbed domains. Vols. I, II, volumes 111, 112 of Operator Theory: Advances and Applications. Birkhäuser Verlag, Basel, 2000.

[27] C. Miranda, Sulle proprietà di regolarità di certe trasformazioni integrali. Atti Accad. Naz. Lincei Mem. Cl. Sci. Fis. Mat. Natur. Sez. I 7 (1965), 303-336.

[28] V. Mityushev and P. M. Adler, Longitudinal permeability of spatially periodic rectangular arrays of circular cylinders. I. A single cylinder in the unit cell. ZAMM Z. Angew. Math. Mech. 82 (2002), 335-345.

[29] P. Musolino, A singularly perturbed Dirichlet problem for the Laplace operator in a periodically perforated domain. A functional analytic approach. Math. Methods Appl. Sci., (to appear).

[30] S. Ozawa, Electrostatic capacity and eigenvalues of the Laplacian. J. Fac. Sci. Univ. Tokyo Sect. IA Math. 30 (1983), 53-62.

[31] L. Preciso, Perturbation analysis of the conformal sewing problem and related problems. PhD Dissertation, University of Padova, 1998.

[32] L. Preciso, Regularity of the composition and of the inversion operator and perturbation analysis of the conformal sewing problem in Roumieu type spaces. Tr. Inst. Mat. Minsk 5 (2000), 99-104.

[33] G. Prodi and A. Ambrosetti, Analisi non lineare. I quaderno. Editrice Tecnico Scientifica, Pisa, 1973.

[34] S. Rogosin, M. Dubatovskaya, and E. Pesetskaya, Eisenstein sums and functions and their application at the study of heat conduction in composites. Siauliai Math. Semin. 4 (2009), 167-187.

[35] G. M. Troianiello, Elliptic differential equations and obstacle problems. The University Series in Mathematics. Plenum Press, New York, 1987.

[36] M. J. Ward and J. B. Keller, Strong localized perturbations of eigenvalue problems. SIAM J. Appl. Math. 53 (1993), 770-798. 